\title{
FULL DISCRETIZATION OF THE POROUS MEDIUM/FAST DIFFUSION EQUATION BASED ON ITS VERY WEAK FORMULATION*
}

\author{
ETIENNE EMMRICH ${ }^{\dagger}$ AND DAVID ŠIŠKA
}

\begin{abstract}
The very weak formulation of the porous medium/fast diffusion equation yields an evolution problem in a Gelfand triple with the pivot space $H^{-1}$. This allows to employ methods of the theory of monotone operators in order to study fully discrete approximations combining a Galerkin method (including conforming finite element methods) with the backward Euler scheme. Convergence is shown even for rough initial data and right-hand sides. The theoretical results are illustrated, in the one-dimensional case, for the piecewise constant finite element approximation of the porous medium equation with the $\delta$-distribution as initial value. As a byproduct, $L^{p}$-stability of the $H^{-1}$-orthogonal projection onto the space of piecewise constant functions is shown for the one-dimensional case.
\end{abstract}

Key words. Porous medium equation, fast diffusion equation, very weak solution, time discretization, Galerkin method, monotone operator, convergence, rough initial data.

AMS subject classifications. 65M12, 35K65, 76S05, 47J35, 47H05.

\section{Introduction}

We will study fully discrete approximations to a class of nonlinear second order degenerate parabolic PDEs including the porous medium equation, heat equation, and fast diffusion equation. Consider

$$
u_{t}-\Delta\left(|u|^{p-2} u\right)=f, \quad p>1, \quad u(\cdot, 0)=u_{0}, \quad u=u(x, t), \quad(x, t) \in \Omega \times(0, T),
$$

where $\Omega \subset \mathbb{R}^{d}$ is a bounded domain of class $\mathscr{C}^{1,1},-\Delta$ is the Laplace operator acting on the spatial variables, $u_{0}$ is a given initial datum, $f$ is a given right-hand side, and we assume some boundary condition on the boundary of $\Omega$. We immediately see that for $p=2$ this is the classical heat equation. For $p>2$, it is referred to as the porous medium equation. For $1<p<2$, the equation above is known as the fast diffusion equation.

The porous medium equation has many applications in natural sciences. As the name suggests, it can model the flow of gas through a porous medium but it also models nonlinear heat transfer, groundwater flow (Boussinesq's model), population dynamics, and a thin liquid film spreading under gravity. It can be used for contour enhancement in image processing. For further applications and more details, see Vázquez [23, Chapters 2 and 21].

As in the case of the heat equation, symmetry arguments can be used to derive exact solutions. We mention, in particular, the Barenblatt solution: an exact solution when the initial condition is Dirac mass at zero. For $p>2$, in the one-dimensional case, the solution is given by (5.2). We will later use this in numerical experiments. The solution is also known in higher dimensions; see again Vázquez [23, Chapter 4]. Jin.

*Received: July 31, 2011; accepted (in revised form): January 2, 2012. Communicated by Shi

This work was supported by Collaborative Research Center 701 "Spectral Structures and Topological Methods in Mathematics", which is funded by DFG (German Research Council).

${ }^{\dagger}$ TU Berlin, Institut für Mathematik, Straße des 17. Juni 136, 10623 Berlin, Germany (emmrich@math.tu-berlin.de).

${ }^{\ddagger}$ Universität Bielefeld, Fakultät für Mathematik, Universitätsstraße 25, 33615 Bielefeld, Germany (dsiska@math.uni-bielefeld.de). 
Throughout the article, we focus on the following generalization of the above equation. For given data $f, g, u_{0}$, we consider the initial-boundary value problem

$$
\left\{\begin{array}{l}
u_{t}-\Delta \alpha(u)=f \text { in } \Omega \times(0, T), \\
\alpha(u)=g \text { on } \partial \Omega \times(0, T), u(\cdot, 0)=u_{0} \text { in } \Omega,
\end{array}\right.
$$

where $\Omega \subset \mathbb{R}^{d}(d \geq 1)$ is assumed to be a bounded domain of class $\mathscr{C}^{1,1}$.

With respect to the function $\alpha: \mathbb{R} \rightarrow \mathbb{R}$, we assume that $\alpha$ is continuous, monotonically increasing, coercive, and fulfills a certain growth condition. In particular, there are numbers $p>1, c>0, \mu>0, \lambda \geq 0$ such that for all $z \in \mathbb{R}$

$$
|\alpha(z)| \leq c\left(|z|^{p-1}+1\right), \quad \alpha(z) z \geq \mu|z|^{p}-\lambda .
$$

Often, $\alpha$ is supposed to fulfill the stronger monotonicity relation (sometimes called "d-monotonicity"; see Gajewski et al. [11, Chapter 3, Definition 1.2])

$$
(\alpha(y)-\alpha(z))(y-z) \geq \mu\left(|y|^{p-1}-|z|^{p-1}\right)(|y|-|z|), \quad y, z \in \mathbb{R},
$$

from which strict monotonicity as well as coercivity follow. The even stronger assumption

$$
(\alpha(y)-\alpha(z))(y-z) \geq \mu|y-z|^{p}, \quad y, z \in \mathbb{R}
$$

leads to uniform monotonicity. Note, however, that (1.4) requires $p \geq 2$.

By taking $\alpha(z)=\int_{0}^{z} \psi(y) d y$, one arrives at (1.1) from the degenerate nonlinear diffusion equation

$$
u_{t}-\nabla \cdot(\psi(u) \nabla u)=f
$$

A typical example is $\alpha(z)=|z|^{p-2} z$ with $\psi(z)=(p-1)|z|^{p-2}$ for $p>1$. For this standard example, relation (1.4) is fulfilled with $\mu=2^{-(p-2)}$ if $p \geq 2$.

For sufficiently smooth functions $u=u(x, t)$ and arbitrary $\tilde{v}=\tilde{v}(x)$ vanishing on the boundary $\partial \Omega$, we obtain, from (1.1) by multiplying with $\tilde{v}$ and carrying out integration by parts twice,

$$
\frac{d}{d t} \int_{\Omega} u \tilde{v} d x-\int_{\Omega} \alpha(u) \Delta \tilde{v} d x=\int_{\Omega} f \tilde{v} d x-\int_{\partial \Omega} g \partial_{\nu} \tilde{v} d \Gamma
$$

where $\partial_{\nu} \equiv \nu \cdot \nabla$ denotes the derivative in the direction of the outer normal $\nu$ on $\partial \Omega$. If $\tilde{v}=:(-\Delta)^{-1} v$ is the solution to the homogeneous Dirichlet problem for the Poisson equation,

$$
-\Delta \tilde{v}=v \text { in } \Omega, \tilde{v}=0 \text { on } \partial \Omega
$$

we formally arrive at

$$
\frac{d}{d t} \int_{\Omega} u(-\Delta)^{-1} v d x+\int_{\Omega} \alpha(u) v d x=\int_{\Omega} f(-\Delta)^{-1} v d x-\int_{\partial \Omega} g \partial_{\nu}(-\Delta)^{-1} v d \Gamma .
$$

We, finally, interpret the time derivative in the weak sense such that for all $\varphi \in$ $\mathscr{C}_{c}^{\infty}(0, T)$,

$$
\begin{aligned}
& -\int_{0}^{T} \int_{\Omega} u(-\Delta)^{-1} v \varphi^{\prime}(t) d x d t+\int_{0}^{T} \int_{\Omega} \alpha(u) v \varphi(t) d x d t \\
= & \int_{0}^{T} \int_{\Omega} f(-\Delta)^{-1} v \varphi(t) d x d t-\int_{0}^{T} \int_{\partial \Omega} g \partial_{\nu}(-\Delta)^{-1} v \varphi(t) d \Gamma d t,
\end{aligned}
$$


supplemented by the initial condition.

This gives rise to study very weak solutions $u \in L^{p}(\Omega \times(0, T))$ to (1.1), where $\int_{\Omega} u(-\Delta)^{-1} v d x$ is to be interpreted as the $H^{-1}(\Omega)$-inner product (see Lions [14, pp. 191f.] for the case $\alpha(z)=|z|^{p-2} z /(p-1)$ as well as Gajewski et al. [11, pp. 72f.]). The use of $H^{-1}(\Omega)$ as the pivot space has recently also been used for dealing with stochastic versions of the porous medium equation; see Beyn et al. [4].

In this paper, we consider the very weak formulation (1.6) and propose a convergent full discretization combining a piecewise constant finite element approximation with the backward Euler scheme.

To be precise, we firstly show that (1.6) is equivalent to an evolution equation governed by a monotone and coercive operator. Next we consider the full discretization of the abstract evolution equation by combining a Galerkin method with the backward Euler scheme. We prove weak and strong convergence results in this abstract setting. These convergence results apply to the finite element/backward Euler approximation of (1.6). Numerical examples support the theoretical results (see Section $5)$. Let us emphasize that these results are not restricted to the porous medium/fast diffusion equation but in fact apply to any first order evolution equation governed by a monotone and coercive operator.

Note that, to the best knowledge of the authors, this is the first paper in which the analytic framework allows to deal with the $\delta$-distribution as initial datum in the onedimensional case. Despite other work, we concentrate on the question of convergence towards a very weak solution rather than derive error estimates, which always require higher regularity of the exact solution. Note that these convergence results, which apply to the situation of rough problem data, cannot be derived from known error estimates or convergence results.

For the piecewise constant finite element approximation in one spatial dimension, we also establish the stability of the orthogonal projection of $H^{-1}(\Omega)$ onto the finite element space with respect to the $L^{p}(\Omega)$-operator norm. This stability of the projection operator has been studied in other function spaces by several authors; see, e.g., Crouzeix \& Thomée [6] for the stability of the $L^{2}(\Omega)$-orthogonal projection onto finite element spaces in $W^{1, p}(\Omega)$.

Spatial approximations of the porous medium equation have, for instance, been studied by Mizutani et al. [15] using nonlinear semigroup theory in $L^{1}(\Omega)$, where convergence is shown. Furthermore they show that their numerical scheme preserves positivity for initial data in $L^{1}(\Omega)$. Finally, they present results of numerical experiments by using an explicit Euler scheme in time. The analysis in [15] is restricted to the case that $u_{0}$ is continuous on $\bar{\Omega}$, and vanishes at $\partial \Omega$, and where $f=g=0$. These and other results are also presented in a wider context in Fujita et al. [10, Chapter 6 ]. Note that the functional analytic framework based on nonlinear semigroup theory (due to Crandall \& Liggett as well as to Brézis \& Pazy), in general, requires $u_{0} \in L^{1}(\Omega)$ and provides convergence in the $L^{\infty}\left(0, T ; L^{1}(\Omega)\right)$ norm.

Numerical approximations using finite element methods in space and Euler and Runge-Kutta methods in time have also been studied previously by others; see $[2,7,17,19]$. For two-dimensional problems with rather regular initial data $u_{0} \in L^{1}(\Omega)$ such that $\alpha\left(u_{0}\right) \in W^{2,1}(\Omega) \cap H_{0}^{1}(\Omega)$, Rulla \& Walkington [21] derive an optimal convergence rate of order $h+\tau$ (for space mesh size $h$ and time step size $\tau$, up to a logarithmic factor) in the norm of $L^{\infty}\left(0, T ; H^{-1}(\Omega)\right)$, using nonlinear semigroup theory, for discretizations based on continuous piecewise linear finite elements and the implicit Euler method. 
The time and space approximation of the porous medium equation (not covering the fast diffusion equation and again with $f=g=0$ ) has recently also been studied in Hansen \& Ostermann [13]. Again for rather regular initial data $u_{0} \in L^{\infty}(\Omega)$ such that $\alpha\left(u_{0}\right) \in W^{2,1}(\Omega)$, they prove an error estimate for Runge-Kutta methods. The estimate is proved, pointwise in $t$, in the norm of the dual of the space of the finite element approximation of $H_{0}^{1}(\Omega)$, which is a very weak norm. In order to come up with a usual (negative) Sobolev norm, one would have to prove stability of an appropriate orthogonal projection onto the finite element space (as is done in this paper in Section 4).

The functional framework with $H^{-1}(\Omega)$ as the pivot space, which is employed in this article, allows us to apply methods and results known for evolution problems governed by monotone operators.

For abstract evolution problems with monotone operators, results on the convergence of the Galerkin method can already be found in the monographs Gajewski et al. [11] and Zeidler [25] and the references cited therein.

The convergence of the Rothe method, i.e., the implicit Euler method, for the approximation of evolution problems governed by (pseudo-) monotone operators has been dealt with, e.g., in the monograph Roubíček [20]. In Emmrich [8], convergence as well as stability and error estimates are provided for the two-step BDF applied to evolution problems governed by monotone operators with a strongly continuous perturbation; see also, e.g., Emmrich \& Thalhammer [9] for Runge-Kutta methods.

The paper is organized as follows: In Section 2, the notation is introduced, the definition of the very weak solution is presented and it is shown that if the very weak solution has sufficient regularity then it is a classical solution. In Section 3, weak and strong convergence results for the full discretization of the abstract problem (2.6) are derived under rather general assumptions. In Section 4, a particular conforming finite element approximation is studied. This is then used in Section 5 for numerical experiments supporting the theoretical results.

\section{Continuous problem}

Throughout this paper, we assume $p \in \Pi$ where $\Pi=(1, \infty)$ for $d \in\{1,2\}$ and $\Pi=[2 d /(d+2), \infty)$ for $d \geq 3$. Moreover, we denote by $q=p /(p-1)$ the exponent conjugated to $p$. Note that $p \geq 2$ refers indeed to the generalized porous medium equation whereas $p<2$ corresponds to the fast diffusion equation.

We employ the standard notation for Lebesgue, Sobolev, Bochner-Lebesgue spaces, and spaces of continuous functions. In particular, we denote by $\|\cdot\|_{m, p, D}$ (resp. $|\cdot|_{m, p, D}$ ) the usual norm (resp. seminorm) in $W^{m, p}(D)(p \in[1, \infty], m \in \mathbb{R})$, and we omit the subscript $D$ if $D=\Omega$. The $L^{2}(\Omega)$-inner product is denoted by $(\cdot, \cdot)_{0,2}$, the usual $H_{0}^{1}(\Omega)$-inner product by $(\cdot, \cdot)_{1,2}=(\nabla \cdot, \nabla \cdot)_{0,2}$.

The dual $V^{*}$ of a Banach space $V$ is equipped with the usual norm $\|f\|_{V^{*}}:=$ $\sup _{v \in V \backslash\{0\}}\langle f, v\rangle_{V^{*} \times V} /\|v\|_{V}$, where $\langle\cdot, \cdot\rangle_{V^{*} \times V}$ denotes the duality pairing.

By $c$, we denote a generic positive constant.

In what follows, let $V:=L^{p}(\Omega)$ with $\|\cdot\|_{V}:=\|\cdot\|_{0, p}$ and $H:=H^{-1}(\Omega):=\left(H_{0}^{1}(\Omega)\right)^{*}$ with $\|\cdot\|_{H}:=\|\cdot\|_{-1,2}$. Denoting by $\tilde{v}=(-\Delta)^{-1} v \in H_{0}^{1}(\Omega)$ the unique weak solution to (1.5) for the right-hand side $v \in H^{-1}(\Omega)$, we have that $(-\Delta)^{-1}: H^{-1}(\Omega) \rightarrow H_{0}^{1}(\Omega)$ is linear and bijective with $\left|(-\Delta)^{-1} v\right|_{1,2}=\|v\|_{-1,2}$ for all $v \in H^{-1}(\Omega)$. This shows that $H$ is a Hilbert space with the inner product

$$
(v, w)_{H}=\frac{1}{4}\left(\|v+w\|_{H}^{2}-\|v-w\|_{H}^{2}\right)
$$




$$
\begin{aligned}
& =\frac{1}{4}\left(\left|(-\Delta)^{-1}(v+w)\right|_{1,2}^{2}-\left|(-\Delta)^{-1}(v-w)\right|_{1,2}^{2}\right) \\
& =\left((-\Delta)^{-1} v,(-\Delta)^{-1} w\right)_{1,2} \\
& =\left\langle v,(-\Delta)^{-1} w\right\rangle_{H^{-1}(\Omega) \times H_{0}^{1}(\Omega)}, \quad v, w \in H .
\end{aligned}
$$

Since $H_{0}^{1}(\Omega) \subset L^{q}(\Omega)$ for $p \in \Pi$, the integral $\int_{\Omega} v w d x$ is well-defined for all $v \in V$, $w \in H_{0}^{1}(\Omega)$. The continuous embedding $H_{0}^{1}(\Omega) \hookrightarrow L^{q}(\Omega)$ then shows that, for any $v \in V$, the mapping $w \mapsto \int_{\Omega} v w d x$ is linear and bounded on $H_{0}^{1}(\Omega)$ and, thus, $j_{1}: V \rightarrow H$ with

$$
\left\langle j_{1}(v), w\right\rangle_{H^{-1}(\Omega) \times H_{0}^{1}(\Omega)}=\int_{\Omega} v w d x, \quad v \in V, w \in H_{0}^{1}(\Omega),
$$

is a well-defined linear bounded injection. This proves the continuous embedding $V \stackrel{j_{1}}{\hookrightarrow} H$. Moreover, the image $j_{1}(V)$ is dense in $H$ and $V$ is reflexive. Then, we also have the continuous and dense embedding $H^{*} \stackrel{j_{-1}}{\longrightarrow} V^{*}$, where $j_{-1}$ is the dual of $j_{1}$ (see Wloka [24, pp. 261f.]). Furthermore, we can identify $H$ and $H^{*}$ by means of the Riesz isomorphism $j_{0}: H \rightarrow H^{*}$ which is given here by

$$
\left\langle j_{0}(v), w\right\rangle_{H^{*} \times H}=\left\langle w,(-\Delta)^{-1} v\right\rangle_{H^{-1}(\Omega) \times H_{0}^{1}(\Omega)}, \quad v, w \in H ;
$$

see also (2.1). So, we come up with the Gelfand triple $V \stackrel{j_{1}}{\longrightarrow} H \stackrel{j_{0}}{=} H^{*} \stackrel{j_{-1}}{\longrightarrow} V^{*}$.

In view of the properties of $\alpha$ (see (1.2)), the integral $\int_{\Omega} \alpha(v) w d x$ is well-defined for all $v, w \in V$ and

$$
\left|\int_{\Omega} \alpha(v) w d x\right| \leq c\left(\|v\|_{V}^{p-1}+1\right)\|w\|_{V}
$$

see also (1.2). This shows that, for any $v \in V$, the mapping $w \mapsto \int_{\Omega} \alpha(v) w d x$ is linear and bounded on $V$. With $\alpha$, we can thus associate the operator $A: V \rightarrow V^{*}$, defined via

$$
\langle A v, w\rangle_{V^{*} \times V}=\int_{\Omega} \alpha(v) w d x, \quad v, w \in V .
$$

The operator $A: V \rightarrow V^{*}$ then is continuous (see Zeidler [25, pp. 561f.]), monotone, and, in view of (1.2), bounded with

$$
\|A v\|_{V^{*}} \leq c\left(\|v\|_{V}^{p-1}+1\right), \quad v \in V
$$

We further have the coercivity relation

$$
\langle A v, v\rangle_{V^{*} \times V} \geq \mu\|v\|_{V}^{p}-\lambda|\Omega|, \quad v \in V .
$$

If $\alpha$ fulfills (1.3) then Hölder's inequality yields

$$
\langle A v-A w, v-w\rangle_{V^{*} \times V} \geq \mu\left(\|v\|_{V}^{p-1}-\|w\|_{V}^{p-1}\right)\left(\|v\|_{V}-\|w\|_{V}\right), \quad v, w \in V,
$$

which implies strict monotonicity as well as coercivity of $A: V \rightarrow V^{*}$ since $V$ is uniformly convex (see Gajewski et al. [11, pp. 62f.]). Under the stronger assumption (1.4), the operator $A: V \rightarrow V^{*}$ is uniformly monotone such that

$$
\langle A v-A w, v-w\rangle_{V^{*} \times V} \geq \mu\|v-w\|_{V}^{p} .
$$


Via $(A u)(t):=A u(t)$, the operator $A: V \rightarrow V^{*}$ can be extended to an operator $A: L^{p}(0, T ; V) \rightarrow\left(L^{p}(0, T ; V)\right)^{*} \equiv L^{q}\left(0, T ; V^{*}\right)$. Note that $L^{p}(0, T ; V)=L^{p}(\Omega \times(0, T))$.

The duality pairing between $L^{p}(0, T ; V)$ and $L^{q}\left(0, T ; V^{*}\right)=\left(L^{p}(0, T ; V)\right)^{*}$ is given by

$$
\langle f, v\rangle_{L^{q}\left(0, T ; V^{*}\right) \times L^{p}(0, T ; V)}=\int_{0}^{T}\langle f(t), v(t)\rangle_{V^{*} \times V} d t .
$$

The solution to our problem is now sought in the Banach space

$$
\mathscr{W}=\left\{v \in \mathscr{X}: v^{\prime} \in \mathscr{X}^{*}\right\}, \quad\|v\|_{\mathscr{W}}=\|v\|_{\mathscr{X}}+\left\|v^{\prime}\right\|_{\mathscr{X}^{*}},
$$

with $v^{\prime}$ being the distributional time derivative and where

$$
\mathscr{X}=L^{p}(0, T ; V) \cap L^{2}(0, T ; H), \quad\|v\|_{\mathscr{X}}=\|v\|_{L^{p}(0, T ; V)}+\|v\|_{L^{2}(0, T ; H)},
$$

is a reflexive, separable Banach space. Its dual $\mathscr{X}^{*}$ can be identified with the sum $L^{q}\left(0, T ; V^{*}\right)+L^{2}(0, T ; H)$, equipped with the norm

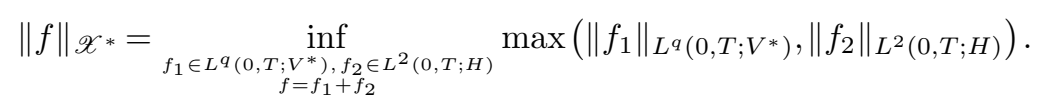

The duality pairing between $f=f_{1}+f_{2} \in L^{q}\left(0, T ; V^{*}\right)+L^{2}(0, T ; H)$ and $v \in \mathscr{X}$ is given by

$$
\langle f, v\rangle_{\mathscr{X} * \times \mathscr{X}}=\int_{0}^{T}\left(\left\langle f_{1}(t), v(t)\right\rangle_{V^{*} \times V}+\left(f_{2}(t), v(t)\right)_{H}\right) d t=\int_{0}^{T}\langle f(t), v(t)\rangle_{V^{*} \times V} d t
$$

see, e.g., Gajewski et al. [11] for more details. Note that $\mathscr{X} \subseteq L^{2}(0, T ; H) \subseteq \mathscr{X}^{*}$ forms a Gelfand triple again and that $\mathscr{W}$ is continuously embedded in $\mathscr{C}([0, T] ; H)$. If $p \geq 2$ then we can just take $\mathscr{X}=L^{p}(0, T ; V), \mathscr{X}^{*}=L^{q}\left(0, T ; V^{*}\right)$.

For what follows, let $W:=W^{2, p}(\Omega) \cap W_{0}^{1, p}(\Omega)$ and $f \in L^{q}\left(0, T ; W^{*}\right)$. Due to the famous result by Agmon-Douglis-Nirenberg, there is for each $v \in V$ a unique solution $(-\Delta)^{-1} v \in W$ to (1.5) that depends continuously on $v$ (see, e.g., Gilbarg \& Trudinger [12, Thm. 9.15, Lemma 9.17]). Recall here that $\partial \Omega \in \mathscr{C}^{1,1}$. So, the mapping

$$
b_{f}: v \mapsto \int_{0}^{T}\left\langle f(t),(-\Delta)^{-1} v(t)\right\rangle_{W^{*} \times W} d t
$$

is linear and bounded on $L^{p}(0, T ; V)$ and thus an element of $\left(L^{p}(0, T ; V)\right)^{*}$.

With respect to the boundary condition in (1.1), we observe that the trace of the derivative in the direction of the outer normal (still denoted by $\partial_{\nu}$ ) is a linear bounded map of $W$ onto $W^{1 / q, p}(\partial \Omega)$ (see Nečas [16, Thm. 5.5 on p. 99]). For boundary values $g \in L^{q}\left(0, T ;\left(W^{1 / q, p}(\partial \Omega)\right)^{*}\right)$, we thus have that

$$
b_{g}: v \mapsto-\int_{0}^{T}\left\langle g(t), \partial_{\nu}(-\Delta)^{-1} v(t)\right\rangle_{\left(W^{1 / q, p}(\partial \Omega)\right)^{*} \times W^{1 / q, p}(\partial \Omega)} d t
$$

is an element of $\left(L^{p}(0, T ; V)\right)^{*}$.

The initial value problem

$$
u^{\prime}+A u=b \text { in }(0, T), \quad u(0)=u_{0},
$$


with $b=b_{f}+b_{g}$ can thus be seen as a generalization of (1.1), and a solution to (2.6) with $b=b_{f}+b_{g}$ will be called a very weak solution to (1.1). result.

With respect to the solvability of (2.6) (and thus of (1.6)), we have the following

Theorem 2.1 (Well-posedness). For any $b \in \mathscr{X}^{*}, u_{0} \in H$, there is a unique solution $u \in \mathscr{W} \subseteq \mathscr{C}([0, T] ; H)$ to (2.6). The solution $u \in \mathscr{C}([0, T] ; H)$ depends continuously on the data $b \in \mathscr{X}^{*}, u_{0} \in H$.

Since $A: V \rightarrow V^{*}$ fulfills the growth condition (2.2), is continuous, coercive, and monotone, Theorem 2.1 follows directly from Gajewski et al. [11, Satz 1.1 on p. 201, Bsp. 3 on p. 215]; see also Barbu [1, Thm. 2.6 on p. 140f.] (for $p \geq 2$ only), Zeidler [25, Thm. 30.A] (with $\tilde{\mathscr{W}}:=\left\{v \in L^{p}(0, T ; V): v^{\prime} \in L^{q}\left(0, T ; V^{*}\right)\right\}$ instead of $\mathscr{W}$ if $p<2$ ), Roubíček [20, Thm. 8.28] (again with $\mathscr{\mathscr { W }}$ instead of $\mathscr{W}$ if $p<2$ ). The continuous dependence on the data easily follows from stability and a priori estimates. Note that the initial condition makes sense in view of the continuous embedding $\mathscr{W} \hookrightarrow \mathscr{C}([0, T] ; H)$. (For $\alpha(z)=|z|^{p-2} z /(p-1)$, Theorem 2.1 then is in accordance with Lions [14, Thm. 3.1 on p. 192], allowing a slightly more general right-hand side $f$.)

Sufficiently regular very weak solutions to (1.1) are classical solutions as is shown in the following.

Theorem 2.2 (Classical solution). Let the data $u_{0} \in H^{-1}(\Omega), f \in L^{q}\left(0, T ; W^{*}\right)$, and $g \in L^{q}\left(0, T ;\left(W^{1 / q, p}(\partial \Omega)\right)^{*}\right)$ be given by corresponding functions $u_{0} \in \mathscr{C}(\bar{\Omega}), f \in$ $\mathscr{C}(\bar{\Omega} \times[0, T])$, and $g \in \mathscr{C}(\partial \Omega \times[0, T])$, such that

$$
\begin{aligned}
\left\langle u_{0}, w\right\rangle_{H^{-1}(\Omega) \times H_{0}^{1}(\Omega)} & =\int_{\Omega} u_{0}(x) w(x) d x \\
\langle f(t), w\rangle_{W^{*} \times W} & =\int_{\Omega} f(x, t) w(x) d x \quad \forall w \in H_{0}^{1}(\Omega), \\
\left\langle g(t), \partial_{\nu} w\right\rangle_{\left(W^{1 / q, p}(\partial \Omega)\right)^{*} \times W^{1 / q, p}(\partial \Omega)} & =\int_{\partial \Omega} g(x, t) \partial_{\nu} w(x) d \Gamma \quad \forall w \in W, t \in[0, T],
\end{aligned}
$$

Then every very weak solution u to (1.1) with

$$
u \in \mathscr{D}:=\left\{v \in \mathscr{C}(\bar{\Omega} \times[0, T]): \exists v_{t}, \Delta \alpha(v) \in \mathscr{C}(\bar{\Omega} \times[0, T])\right\}
$$

satisfies (1.1) in the classical sense.

Proof. The function $u$ fulfills the initial condition since for all $v \in H_{0}^{1}(\Omega)$

$$
\begin{gathered}
\int_{\Omega} u_{0}(x) v(x) d x=\left\langle u_{0}, v\right\rangle_{H^{-1}(\Omega) \times H_{0}^{1}(\Omega)} \\
=\left\langle j_{1}(u(0)), v\right\rangle_{H^{-1}(\Omega) \times H_{0}^{1}(\Omega)}=\int_{\Omega} u(x, 0) v(x) d x .
\end{gathered}
$$

By density and the properties of functions in $\mathscr{W}$, the evolution equation in (2.6) 
is equivalent to the requirement that for all $v \in V, \phi \in \mathscr{C}_{c}^{1}((0, T))$,

$$
\begin{aligned}
& -\int_{0}^{T}\left(j_{1}(u(\cdot, t)), j_{1}(v)\right)_{H} \phi^{\prime}(t) d t+\int_{0}^{T} \int_{\Omega} \alpha(u(x, t)) v(x) \phi(t) d x d t \\
= & \int_{0}^{T}\left\langle f(t),(-\Delta)^{-1} v\right\rangle_{W^{*} \times W} \phi(t) d t \\
& -\int_{0}^{T}\left\langle g(t), \partial_{\nu}(-\Delta)^{-1} v\right\rangle_{\left(W^{1 / q, p}(\partial \Omega)\right)^{*} \times W^{1 / q, p}(\partial \Omega)} \phi(t) d t \\
= & \int_{0}^{T} \int_{\Omega} f(x, t)(-\Delta)^{-1} v(x) \phi(t) d x d t-\int_{0}^{T} \int_{\partial \Omega} g(x, t) \partial_{\nu}(-\Delta)^{-1} v(x) \phi(t) d \Gamma d t .
\end{aligned}
$$

With (2.1), the definition of $j_{1},(-\Delta)^{-1}$, and $A$, and with integration by parts, we find for almost all $t \in(0, T)$

$$
\left(j_{1}(u(\cdot, t)), j_{1}(v)\right)_{H}=\left((-\Delta)^{-1} u(\cdot, t),(-\Delta)^{-1} v\right)_{1,2}=\int_{\Omega} u(x, t)(-\Delta)^{-1} v(x) d x .
$$

Substituting $(-\Delta)^{-1} v$ by $\tilde{v} \in W$ (remember that $(-\Delta)^{-1}: V \rightarrow W$ is bijective), we obtain with integration by parts

$$
\begin{aligned}
& \int_{0}^{T}\left(\int_{\Omega}\left(u_{t}(x, t)-\Delta \alpha(u(x, t))-f(x, t)\right) \tilde{v}(x) d x\right. \\
& \left.+\int_{\partial \Omega}(g(x, t)-\alpha(u(x, t))) \partial_{\nu} \tilde{v}(x) d \Gamma\right) \phi(t) d t=0,
\end{aligned}
$$

which finally shows that $u$ fulfills (1.1).

\section{Fully discrete approximation and its convergence}

In this section we derive, under rather general assumptions, weak and strong convergence results for the full discretization of the abstract problem (2.6). This abstract setting applies to, but is not restricted to, the very weak formulation of the porous medium/fast diffusion equation.

For $N \in \mathbb{N}$, let $\tau:=T / N, t_{n}:=n \tau(n=0,1, \ldots, N)$. Moreover, let $\left\{V_{m}\right\}_{m \in \mathbb{N}}$ be a Galerkin scheme for the separable Banach space $V$ consisting of finite dimensional subspaces $V_{m}$ with $V_{m} \subseteq V_{m+1}$ that satisfy the property of limited completeness:

$$
\operatorname{clos}_{\|\cdot\|_{V}} \bigcup_{m=1}^{\infty} V_{m}=V .
$$

For given approximations $u^{0} \in V_{m}$ of the initial value and $\left\{b^{n}\right\}_{n=1}^{N} \subset V^{*}$ of the right-hand side, we then look for approximations $\left\{u^{n}\right\}_{n=1}^{N} \subset V_{m}, u^{n} \approx u\left(t_{n}\right)$, of the exact solution to (2.6) such that for all $n=1,2, \ldots, N$,

$$
\left(\frac{u^{n}-u^{n-1}}{\tau}, v\right)_{H}+\left\langle A u^{n}, v\right\rangle_{V^{*} \times V}=\left\langle b^{n}, v\right\rangle_{V^{*} \times V} \quad \forall v \in V_{m} .
$$

Recall that $A: V \rightarrow V^{*}$ is monotone, hemicontinuous, fulfills the growth condition (2.2) and the coercivity condition (2.3). In this abstract setting, we can now derive the following results. 
Theorem 3.1 (Discrete problem and a priori estimates I). For any $u^{0} \in V_{m}$ and $\left\{b^{n}\right\}_{n=1}^{N} \subset V^{*}$ there exists a unique solution $\left\{u^{n}\right\}_{n=1}^{N} \subset V_{m}$ to (3.1). Let $b^{n}=b_{1}^{n}+b_{2}^{n}$ with $b_{1}^{n} \in V^{*}$ and $b_{2}^{n} \in H(n=1,2, \ldots, N)$. Then the discrete solution $\left\{u^{n}\right\}_{n=1}^{N}$ fulfills the a priori estimate

$$
\begin{aligned}
& \max _{n=1,2, \ldots, N}\left\|u^{n}\right\|_{H}^{2}+\sum_{n=1}^{N}\left\|u^{n}-u^{n-1}\right\|_{H}^{2}+\tau \sum_{n=1}^{N}\left\|u^{n}\right\|_{V}^{p} \\
\leq & c\left(\left\|u^{0}\right\|_{H}^{2}+\tau \sum_{n=1}^{N}\left\|b_{1}^{n}\right\|_{V^{*}}^{q}+\tau \sum_{n=1}^{N}\left\|b_{2}^{n}\right\|_{H}^{2}+1\right) .
\end{aligned}
$$

Proof. Existence and uniqueness follow step by step from the Browder-Minty theorem since the operator appearing in each step is $\frac{1}{\tau} I+A$, which is strictly monotone, hemicontinuous and coercive. The a priori estimates can be proved similarly as in Emmrich [8, Sect. 4] by employing the algebraic relation

$$
2(a-b) a=a^{2}-b^{2}+(a-b)^{2}, \quad a, b \in \mathbb{R},
$$

together with the coercivity of $A$, and Young's inequality.

We may also derive an a priori estimate for the discrete time derivative, which later will allow us to apply the Lions-Aubin theorem in order to prove a strong convergence result.

Theorem 3.2 (A priori estimates II). Let $u^{0} \in V_{m}$ and $b^{n}=b_{1}^{n}+b_{2}^{n}$ with $b_{1}^{n} \in V^{*}$ and $b_{2}^{n} \in H(n=1,2, \ldots, N)$. The discrete solution $\left\{u^{n}\right\}_{n=1}^{N} \subset V_{m}$ to (3.1) then fulfills the a priori estimate

$$
\begin{aligned}
& \tau \sum_{n=1}^{N}\left\|\frac{u^{n}-u^{n-1}}{\tau}\right\|_{V^{*}}^{q} \\
\leq & c\left\|P_{m}\right\|_{V \leftarrow V}^{q}\left(\left\|u^{0}\right\|_{H}^{2}+\tau \sum_{n=1}^{N}\left\|b_{1}^{n}\right\|_{V^{*}}^{q}+\tau \sum_{n=1}^{N}\left\|b_{2}^{n}\right\|_{V^{*}}^{q}+\tau \sum_{n=1}^{N}\left\|b_{2}^{n}\right\|_{H}^{2}+1\right) .
\end{aligned}
$$

Here, $\quad P_{m}: H \rightarrow V_{m} \quad(m \in \mathbb{N})$ is the orthogonal projection of $H$ onto $V_{m}$ and $\left\|P_{m}\right\|_{V \leftarrow V}:=\sup _{v \in V \backslash\{0\}}\left\|P_{m} v\right\|_{V} /\|v\|_{V}$ denotes the operator norm of $P_{m}$ as an operator in $V$.

Proof. By definition and inserting the scheme (3.1), we have

$$
\begin{aligned}
\left\|\frac{u^{n}-u^{n-1}}{\tau}\right\|_{V^{*}} & =\sup _{v \in V \backslash\{0\}} \frac{1}{\|v\|_{V}}\left(\frac{u^{n}-u^{n-1}}{\tau}, v\right)_{H} \\
& =\sup _{v \in V \backslash\{0\}} \frac{1}{\|v\|_{V}}\left(\frac{u^{n}-u^{n-1}}{\tau}, P_{m} v\right)_{H} \\
& =\sup _{v \in V \backslash\{0\}} \frac{1}{\|v\|_{V}}\left\langle b^{n}-A u^{n}, P_{m} v\right\rangle_{V^{*} \times V} \\
& \leq\left\|b^{n}-A u^{n}\right\|_{V^{*}}\left\|P_{m}\right\|_{V \leftarrow V} .
\end{aligned}
$$

With the growth condition (2.2) for $A$, we find

$$
\left\|b^{n}-A u^{n}\right\|_{V^{*}} \leq\left\|b^{n}\right\|_{V^{*}}+c\left(\left\|u^{n}\right\|_{V}^{p-1}+1\right)
$$


and thus

$$
\tau \sum_{n=1}^{N}\left\|b^{n}-A u^{n}\right\|_{V^{*}}^{q} \leq c \tau \sum_{n=1}^{N}\left\|b^{n}\right\|_{V^{*}}^{q}+c \tau \sum_{n=1}^{N}\left\|u^{n}\right\|_{V}^{p}+c .
$$

This proves, together with (3.2), the assertion.

The appearance of the term $\tau \sum_{n=1}^{N}\left\|b_{2}^{n}\right\|_{V^{*}}^{q}$ on the right-hand side in (3.4) is no problem if $p \geq 2$ or if $b \in L^{q}\left(0, T ; V^{*}\right)$ (even for $p<2$ ). It could also be avoided if the part $b_{2}$ of the right-hand side $b$ is also approximated in $V_{m}$.

In what follows, we consider a sequence of time grids and finite dimensional approximations of $V$ and prove convergence of the corresponding sequence of numerical solutions.

Let $\left\{m_{\ell}\right\}_{\ell \in \mathbb{N}} \subset \mathbb{N}$ and $\left\{N_{\ell}\right\}_{\ell \in \mathbb{N}} \subset \mathbb{N}$ be two nondecreasing sequences tending to infinity as $\ell \rightarrow \infty$. From the discrete solution $\left\{u^{n}\right\}_{n=0}^{N}$ that corresponds to the partition of $[0, T]$ with the step size $\tau_{\ell}=T / N_{\ell}$ and the finite dimensional space $V_{m_{\ell}}$, we construct the piecewise constant interpolant $u_{\ell}$ with $u_{\ell}(t)=u^{n}$ for $t \in\left(t_{n-1}, t_{n}\right]\left(n=1,2, \ldots, N_{\ell}\right)$, $u_{\ell}(0)=u^{1}$. Moreover, let $\hat{u}_{\ell}$ be the piecewise linear interpolation of the points $\left(t_{n}, u^{n}\right)$ $\left(n=0,1, \ldots, N_{\ell}\right)$. Note that $\hat{u}_{\ell}$ is piecewise differentiable with $\hat{u}_{\ell}^{\prime}(t)=\left(u^{n}-u^{n-1}\right) / \tau_{\ell}$ for $t \in\left(t_{n-1}, t_{n}\right)\left(n=1,2, \ldots, N_{\ell}\right)$. For simplicity, the approximation of the right-hand side shall be given by

$$
b^{n}:=\frac{1}{\tau_{\ell}} \int_{t_{n-1}}^{t_{n}} b(t) d t, \quad n=1,2, \ldots, N_{\ell} .
$$

By $b_{\ell}$, we denote the corresponding piecewise constant interpolant (again taking the values at the right endpoints of the subintervals). For $b=b_{1}+b_{2}$, we also work with the corresponding approximations $b_{1}^{n}, b_{2}^{n}$ and $b_{1, \ell}, b_{2, \ell}$. If $b$ is sufficiently regular, one may also take $b^{n}=b\left(t_{n}\right)$ or any other restriction onto the time grid such that $b_{\ell}$ converges strongly towards $b$ in $\mathscr{X}^{*}$ as $\ell \rightarrow \infty$.

The following convergence results are general results for nonlinear evolution problems governed by a monotone and coercive operator and are not restricted to the current context of the porous medium/fast diffusion equation.

Theorem 3.3 (Weak convergence). Let $u_{0} \in H$ and $b \in \mathscr{X}^{*}$ be given and assume $u_{\ell}^{0} \rightarrow u_{0}$ in $H$ as $\ell \rightarrow \infty$. The sequence of piecewise constant interpolants $u_{\ell}$ corresponding to the discrete solutions to (3.1) then converges weakly in $L^{p}(0, T ; V)$ and weakly* in $L^{\infty}(0, T ; H)$ towards the exact solution $u \in \mathscr{W}$ to (2.6) as $\ell \rightarrow \infty$. The sequence of the corresponding piecewise linear interpolants $\hat{u}_{\ell}$ converges weakly* in $L^{\infty}(0, T ; H)$ towards the exact solution $u$.

Furthermore, if there is a constant $c>0$ such that for all $\ell \in \mathbb{N}$ we have

$$
\tau_{\ell}\left\|u_{\ell}^{0}\right\|_{V}^{p} \leq c
$$

then the sequence of piecewise linear interpolants $\hat{u}_{\ell}$ converges weakly in $L^{p}(0, T ; V)$ towards the exact solution $u$.

Remark 3.4. Assumption (3.6) can always be fulfilled since $V$ is dense in $H$. Depending on the initial datum, however, (3.6) may result in a coupling of the time step and spatial mesh size.

The proof of the preceding convergence result will be prepared by the following preliminary results. 
LEMMA 3.5. Under the assumptions of Theorem 3.3 there is an element $u \in \mathscr{X}$ and a subsequence, denoted by $\ell^{\prime}$, such that

$$
u_{\ell^{\prime}}, \hat{u}_{\ell^{\prime}} \stackrel{*}{\rightarrow} u \text { in } L^{\infty}(0, T ; H), \quad u_{\ell^{\prime}} \rightarrow u \text { in } L^{p}(0, T ; V) \text { as } \ell^{\prime} \rightarrow \infty .
$$

Moreover, there is an element $\xi \in H$ such that

$$
\hat{u}_{\ell^{\prime}}(T) \rightarrow \xi \text { in } H \text { as } \ell^{\prime} \rightarrow \infty
$$

Finally, there is an element $a \in L^{q}\left(0, T ; V^{*}\right)$ such that

$$
A u_{\ell^{\prime}} \rightarrow a \text { in } L^{q}\left(0, T ; V^{*}\right) \text { as } \ell^{\prime} \rightarrow \infty
$$

If (3.6) is fulfilled then also

$$
\hat{u}_{\ell^{\prime}} \rightarrow u \text { in } L^{p}(0, T ; V) \text { as } \ell^{\prime} \rightarrow \infty
$$

Proof. From the corresponding definition, we immediately get

$$
\left\|u_{\ell}\right\|_{L^{\infty}(0, T ; H)}=\max _{n=1,2, \ldots, N_{\ell}}\left\|u^{n}\right\|_{H}, \quad\left\|u_{\ell}\right\|_{L^{p}(0, T ; V)}^{p}=\tau_{\ell} \sum_{n=1}^{N_{\ell}}\left\|u^{n}\right\|_{V}^{p}
$$

as well as

$$
\left\|\hat{u}_{\ell}\right\|_{L^{\infty}(0, T ; H)}=\max _{n=0,1, \ldots, N_{\ell}}\left\|u^{n}\right\|_{H}
$$

Since the sequence $\left\{u_{\ell}^{0}\right\}_{\ell \in \mathbb{N}} \subset H$ is convergent, it is also bounded. As $b \in \mathscr{X}^{*}$, there holds $b=b_{1}+b_{2}$ with $b_{1} \in L^{q}\left(0, T ; V^{*}\right)$ and $b_{2} \in L^{2}(0, T ; H)$. With Hölder's inequality, we find for the approximation (3.5) that

$$
\tau_{\ell} \sum_{n=1}^{N_{\ell}}\left\|b_{1}^{n}\right\|_{V^{*}}^{q} \leq\left\|b_{1}\right\|_{L^{q}\left(0, T ; V^{*}\right)}^{q}, \quad \tau_{\ell} \sum_{n=1}^{N_{\ell}}\left\|b_{2}^{n}\right\|_{H}^{2} \leq\left\|b_{2}\right\|_{L^{2}(0, T ; H)}^{2} .
$$

From the a priori estimate given in Theorem 3.1 , we hence conclude that $\left\{u_{\ell}\right\}_{\ell \in \mathbb{N}}$ is bounded in $L^{\infty}(0, T ; H)$ as well as in $L^{p}(0, T ; V)$. Moreover, $\left\{\hat{u}_{\ell}\right\}_{\ell \in \mathbb{N}}$ is bounded in $L^{\infty}(0, T ; H)$. By standard compactness arguments (see, e.g., Brézis [5, Cor. III.26, Thm. III.27]), we thus have elements $u \in L^{p}(0, T ; V) \cap L^{\infty}(0, T ; H)$ and $\hat{u} \in L^{\infty}(0, T ; H)$ and a subsequence, denoted by $\ell^{\prime}$, such that

$$
u_{\ell^{\prime}} \rightarrow u \text { in } L^{p}(0, T ; V), u_{\ell^{\prime}} \stackrel{*}{\rightarrow} u \text { in } L^{\infty}(0, T ; H), \hat{u}_{\ell^{\prime}} \stackrel{*}{\rightarrow} \hat{u} \text { in } L^{\infty}(0, T ; H)
$$

as $\ell^{\prime} \rightarrow \infty$.

Because of

$$
\left\|\hat{u}_{\ell}-u_{\ell}\right\|_{L^{2}(0, T ; H)}^{2}=\frac{\tau_{\ell}}{3} \sum_{n=1}^{N_{\ell}}\left\|u^{n}-u^{n-1}\right\|_{H}^{2}
$$

and the a priori estimate (3.2), we have the strong convergence

$$
\hat{u}_{\ell}-u_{\ell} \rightarrow 0 \text { in } L^{2}(0, T ; H) \text { as } \ell \rightarrow \infty \text {. }
$$


As the weak* convergence in $L^{\infty}(0, T ; H)$ yields the weak convergence in $L^{2}(0, T ; H)$, $u$ and $\hat{u}$ must coincide.

Again from the a priori estimate (3.2), we have the uniform boundedness of $\hat{u}_{\ell}(T)=u^{N_{\ell}}$ in $H$, which implies the weak convergence of a subsequence.

An immediate consequence of the growth condition (2.2) and the a priori estimate (3.2) is the uniform boundedness of

$$
\left\|A u_{\ell}\right\|_{L^{q}\left(0, T ; V^{*}\right)}^{q} \leq c\left(1+\left\|u_{\ell}\right\|_{L^{p}(0, T ; V)}^{p}\right),
$$

which shows the weak convergence of a subsequence of $\left\{A u_{\ell}\right\}_{\ell \in \mathbb{N}}$ in $L^{q}\left(0, T ; V^{*}\right)$.

Since

$$
\left\|\hat{u}_{\ell}\right\|_{L^{p}(0, T ; V)}^{p} \leq c \tau_{\ell} \sum_{n=0}^{N_{\ell}}\left\|u^{n}\right\|_{V}^{p},
$$

we get with (3.6) also the weak convergence of a subsequence $\hat{u}_{\ell^{\prime}}$ in $L^{p}(0, T ; V)$, and the limit can only be $u$.

Proof. [of Theorem 3.3] We start by rewriting the numerical scheme as

$$
\left\langle\hat{u}_{\ell}^{\prime}(t), v\right\rangle_{V^{*} \times V}+\left\langle A u_{\ell}(t), v\right\rangle_{V^{*} \times V}=\left\langle b_{\ell}(t), v\right\rangle_{V^{*} \times V} \quad \forall v \in V_{m_{\ell}} .
$$

This equation holds pointwise for all $t \in\left(t_{n-1}, t_{n}\right)\left(n=1,2, \ldots, N_{\ell}\right)$ as well as in the distributional sense on $(0, T)$.

Let $k \in \mathbb{N}$ be arbitrary but fixed. Then (3.8) implies that for all $\ell \geq k$

$$
\begin{gathered}
-\int_{0}^{T}\left(\hat{u}_{\ell}(t), v\right)_{H} \phi^{\prime}(t) d t+\int_{0}^{T}\left\langle A u_{\ell}(t), v\right\rangle_{V^{*} \times V} \phi(t) d t=\int_{0}^{T}\left\langle b_{\ell}(t), v\right\rangle_{V^{*} \times V} \phi(t) d t \\
\forall v \in V_{m_{k}}, \phi \in \mathscr{C}_{c}^{\infty}(0, T)
\end{gathered}
$$

since, in particular, $V_{m_{k}} \subseteq V_{m_{\ell}}$. In view of Lemma 3.5 and since (by standard arguments)

$$
b_{\ell} \rightarrow b \text { in } \mathscr{X}^{*} \text { as } \ell \rightarrow \infty,
$$

we find in the limit, passing to a subsequence (still denoted by $\ell$ ) if necessary,

$$
\begin{gathered}
-\int_{0}^{T}(u(t), v)_{H} \phi^{\prime}(t) d t+\int_{0}^{T}\langle a(t), v\rangle_{V^{*} \times V} \phi(t) d t=\int_{0}^{T}\langle b(t), v\rangle_{V^{*} \times V} \phi(t) d t \\
\forall v \in V_{m_{k}}, \phi \in \mathscr{C}_{c}^{\infty}(0, T) .
\end{gathered}
$$

Because of the limited completeness of the Galerkin scheme and the definition of the weak derivative, this shows that $u \in \mathscr{X}$ possesses the weak derivative

$$
u^{\prime}=b-a \in \mathscr{X}^{*} \text {. }
$$

Therefore, we also have $u \in \mathscr{W}$, and it remains to prove $u(0)=u_{0}$ as well as $a=A u$.

Let $k \in \mathbb{N}$ be arbitrary but fixed. Then, for all $\ell \geq k$, we observe the following: Since $u, \hat{u}_{\ell} \in \mathscr{W}$, we can employ integration by parts and obtain for all $v \in V_{m_{k}}$ and $\phi \in \mathscr{C}^{1}([0, T])$ that

$$
\begin{aligned}
& (u(T), v)_{H} \phi(T)-(u(0), v)_{H} \phi(0) \\
= & \int_{0}^{T}\left(\left\langle u^{\prime}(t), v\right\rangle_{V^{*} \times V} \phi(t)+\langle u(t), v\rangle_{V^{*} \times V} \phi^{\prime}(t)\right) d t \\
= & \int_{0}^{T}\left(\langle b(t)-a(t), v\rangle_{V^{*} \times V} \phi(t)+\langle u(t), v\rangle_{V^{*} \times V} \phi^{\prime}(t)\right) d t
\end{aligned}
$$




$$
\begin{gathered}
=\int_{0}^{T}\left(\left\langle b(t)-b_{\ell}(t)+\hat{u}_{\ell}^{\prime}(t)+A u_{\ell}(t)-a(t), v\right\rangle_{V^{*} \times V} \phi(t)+\langle u(t), v\rangle_{V^{*} \times V} \phi^{\prime}(t)\right) d t \\
=\int_{0}^{T}\left(\left\langle b(t)-b_{\ell}(t)+A u_{\ell}(t)-a(t), v\right\rangle_{V^{*} \times V} \phi(t)+\left\langle u(t)-\hat{u}_{\ell}(t), v\right\rangle_{V^{*} \times V} \phi^{\prime}(t)\right) d t \\
+\left(\hat{u}_{\ell}(T), v\right)_{H} \phi(T)-\left(\hat{u}_{\ell}(0), v\right)_{H} \phi(0) .
\end{gathered}
$$

Taking the limit on the right-hand side and recalling $\hat{u}_{\ell}(0)=u_{\ell}^{0} \rightarrow u_{0}$ in $H$, we conclude that

$$
(u(T), v)_{H} \phi(T)-(u(0), v)_{H} \phi(0)=(\xi, v)_{H} \phi(T)-\left(u_{0}, v\right)_{H} \phi(0) \quad \forall v \in V_{m_{k}} .
$$

Choosing $\phi(T)=0$ and $\phi(0)=0$, respectively, we find that $u(0)=u_{0}$ and $u(T)=\xi$ in $H$ due to the limited completeness of the Galerkin scheme in $V$ and the density of $V$ in $H$.

From (3.8), we find

$$
\int_{0}^{T}\left\langle\hat{u}_{\ell}^{\prime}(t), u_{\ell}(t)\right\rangle_{V^{*} \times V} d t+\int_{0}^{T}\left\langle A u_{\ell}(t), u_{\ell}(t)\right\rangle_{V^{*} \times V} d t=\int_{0}^{T}\left\langle b_{\ell}(t), u_{\ell}(t)\right\rangle_{V^{*} \times V} d t
$$

For the first term, a short calculation employing (3.3) shows that

$$
\begin{aligned}
\int_{0}^{T}\left\langle\hat{u}_{\ell}^{\prime}(t), u_{\ell}(t)\right\rangle_{V^{*} \times V} d t & =\sum_{n=1}^{N_{\ell}} \int_{t_{n-1}}^{t_{n}}\left(\frac{u^{n}-u^{n-1}}{\tau}, u^{n}\right)_{H} d t \\
& =\frac{1}{2} \sum_{n=1}^{N_{\ell}}\left(\left\|u^{n}\right\|_{H}^{2}-\left\|u^{n-1}\right\|_{H}^{2}+\left\|u^{n}-u^{n-1}\right\|_{H}^{2}\right) \\
& \geq \frac{1}{2}\left\|u^{N_{\ell}}\right\|_{H}^{2}-\frac{1}{2}\left\|u_{\ell}^{0}\right\|_{H}^{2} .
\end{aligned}
$$

Upon noting that $u^{N_{\ell}}=\hat{u}_{\ell}(T)$, the weak convergence of $\hat{u}_{\ell}(T)$ towards $\xi=u(T)$ in $H$, together with the weak lower semicontinuity of the norm, and the strong convergence of $u_{\ell}^{0}$ towards $u_{0}=u(0)$ in $H$ yields, together with an integration by parts,

$$
\int_{0}^{T}\left\langle u^{\prime}(t), u(t)\right\rangle_{V^{*} \times V} d t=\frac{1}{2}\|u(T)\|_{H}^{2}-\frac{1}{2}\left\|u_{0}\right\|_{H}^{2} \leq \liminf _{\ell \rightarrow \infty} \int_{0}^{T}\left\langle\hat{u}_{\ell}^{\prime}(t), u_{\ell}(t)\right\rangle_{V^{*} \times V} d t .
$$

For the second term on the left-hand side in (3.9), we employ the monotonicity of $A$. For arbitrary $w \in L^{p}(0, T ; V)$, we have

$$
\begin{aligned}
& \int_{0}^{T}\left\langle A u_{\ell}(t), u_{\ell}(t)\right\rangle_{V^{*} \times V} d t \\
\geq & \int_{0}^{T}\left(\left\langle A u_{\ell}(t), u_{\ell}(t)\right\rangle_{V^{*} \times V} d t-\left\langle A u_{\ell}(t)-A w(t), u_{\ell}(t)-w(t)\right\rangle_{V^{*} \times V}\right) d t \\
= & \int_{0}^{T}\left\langle A u_{\ell}(t), w(t)\right\rangle_{V^{*} \times V} d t+\int_{0}^{T}\left\langle A w(t), u_{\ell}(t)-w(t)\right\rangle_{V^{*} \times V} d t .
\end{aligned}
$$

In the limit, we thus come up with

$$
\liminf _{\ell \rightarrow \infty} \int_{0}^{T}\left\langle A u_{\ell}(t), u_{\ell}(t)\right\rangle_{V^{*} \times V} d t
$$




$$
\geq \int_{0}^{T}\langle a(t), w(t)\rangle_{V^{*} \times V} d t+\int_{0}^{T}\langle A w(t), u(t)-w(t)\rangle_{V^{*} \times V} d t .
$$

Because of the strong convergence of $b_{\ell}$ towards $b$ in $\mathscr{X}^{*}$ and the weak convergence of $u_{\ell}$ towards $u$ in $\mathscr{X}$, we now obtain from (3.9)

$$
\begin{aligned}
& \int_{0}^{T}\left\langle u^{\prime}(t)+a(t), u(t)\right\rangle_{V^{*} \times V} d t=\int_{0}^{T}\langle b(t), u(t)\rangle_{V^{*} \times V} d t=\lim _{\ell \rightarrow \infty} \int_{0}^{T}\left\langle b_{\ell}(t), u_{\ell}(t)\right\rangle_{V^{*} \times V} d t \\
= & \lim _{\ell \rightarrow \infty}\left(\int_{0}^{T}\left\langle\hat{u}_{\ell}^{\prime}(t), u_{\ell}(t)\right\rangle_{V^{*} \times V} d t+\int_{0}^{T}\left\langle A u_{\ell}(t), u_{\ell}(t)\right\rangle_{V^{*} \times V} d t\right) \\
\geq & \int_{0}^{T}\left\langle u^{\prime}(t), u(t)\right\rangle_{V^{*} \times V} d t+\int_{0}^{T}\langle a(t), w(t)\rangle_{V^{*} \times V} d t+\int_{0}^{T}\langle A w(t), u(t)-w(t)\rangle_{V^{*} \times V} d t .
\end{aligned}
$$

This leads to

$$
\int_{0}^{T}\langle A w(t), w(t)-u(t)\rangle_{V^{*} \times V} d t \geq \int_{0}^{T}\langle a(t), w(t)-u(t)\rangle_{V^{*} \times V} d t
$$

and the hemicontinuity of $A$ shows that $A u=a$ in $L^{q}\left(0, T ; V^{*}\right)$ such that

$$
u^{\prime}+A u=b \text { in } \mathscr{X}^{*} .
$$

Under the additional assumption (3.6), we obtain, in view of Lemma 3.5, the additional convergence of the piecewise linear interpolants in time. Finally, by contradiction, we may prove that the whole sequence (and not only a subsequence) converges because of the uniqueness of the exact solution.

Under additional assumptions, we may also prove strong convergence results. We first derive a result of strong convergence if the governing operator fulfills a stronger monotonicity assumption (without requiring additional compactness of the embedding of $V$ in $H$ ).

TheOREM 3.6 (Strong convergence I). Let $u_{0} \in H$ and $b \in \mathscr{X}^{*}$ be given, assume $u_{\ell}^{0} \rightarrow u_{0}$ in $H$ as $\ell \rightarrow \infty$ and let $A: V \rightarrow V^{*}$ satisfy the stronger monotonicity assumption (2.4) or even (2.5). Then, in addition to the assertions of Theorem 3.3, $u_{\ell}$ converges strongly in $L^{p}(0, T ; V)$ towards the exact solution. Moreover, $u_{\ell}$ and $\hat{u}_{\ell}$ converge strongly in $L^{r}(0, T ; H)$ for any $r \in[1, \infty)$ towards the exact solution $u$.

Proof. We firstly observe with Hölder's inequality that (2.4) implies for any $v, w \in \mathscr{X}$

$$
\begin{gathered}
\int_{0}^{T}\langle A v(t)-A w(t), v(t)-w(t)\rangle_{V^{*} \times V} d t \\
\geq \mu\left(\|v\|_{L^{p}(0, T ; V)}^{p-1}-\|w\|_{L^{p}(0, T ; V)}^{p-1}\right)\left(\|v\|_{L^{p}(0, T ; V)}-\|w\|_{L^{p}(0, T ; V)}\right) .
\end{gathered}
$$

Hence, we find with (3.9)

$$
\begin{aligned}
0 & \leq \mu\left(\left\|u_{\ell}\right\|_{L^{p}(0, T ; V)}^{p-1}-\|u\|_{L^{p}(0, T ; V)}^{p-1}\right)\left(\left\|u_{\ell}\right\|_{L^{p}(0, T ; V)}-\|u\|_{L^{p}(0, T ; V)}\right) \\
& \leq \int_{0}^{T}\left\langle A u_{\ell}(t)-A u(t), u_{\ell}(t)-u(t)\right\rangle_{V^{*} \times V} d t
\end{aligned}
$$




$$
\begin{aligned}
= & \int_{0}^{T}\left\langle b_{\ell}(t), u_{\ell}(t)\right\rangle_{V^{*} \times V} d t-\int_{0}^{T}\left\langle\hat{u}_{\ell}^{\prime}(t), u_{\ell}(t)\right\rangle_{V^{*} \times V} d t \\
& -\int_{0}^{T}\left\langle A u_{\ell}(t), u(t)\right\rangle_{V^{*} \times V} d t-\int_{0}^{T}\left\langle A u(t), u_{\ell}(t)-u(t)\right\rangle_{V^{*} \times V} d t .
\end{aligned}
$$

Because of (3.10) and (3.11), the limit superior of the right-hand side of the foregoing estimate can be estimated from above by

$$
\int_{0}^{T}\langle b(t), u(t)\rangle_{V^{*} \times V} d t-\int_{0}^{T}\left\langle u^{\prime}(t), u(t)\right\rangle_{V^{*} \times V} d t-\int_{0}^{T}\langle A u(t), u(t)\rangle_{V^{*} \times V} d t=0 .
$$

This shows that

$$
\left\|u_{\ell}\right\|_{L^{p}(0, T ; V)} \rightarrow\|u\|_{L^{p}(0, T ; V)} \text { as } \ell \rightarrow \infty .
$$

Since $L^{p}(0, T ; V)$ is uniformly convex and since $u_{\ell}$ converges already weakly towards $u$ in $L^{p}(0, T ; V)$, this implies (see, e.g., Brézis [5, Prop. III.30]) the strong convergence of $u_{\ell}$ towards $u$ in $L^{p}(0, T ; V)$.

In case of the even stronger assumption (2.5) of uniform monotonicity, we immediately have

$$
\mu\left\|u_{\ell}-u\right\|_{L^{p}(0, T ; V)}^{p} \leq \int_{0}^{T}\left\langle A u_{\ell}(t)-A u(t), u_{\ell}(t)-u(t)\right\rangle_{V^{*} \times V} d t
$$

and the proof above applies again.

Since $V \hookrightarrow H$, the strong convergence in $L^{p}(0, T ; V)$ also implies the strong convergence in $L^{p}(0, T ; H)$. Since $\left\{u_{\ell}\right\}$ is bounded in $L^{\infty}(0, T ; H)$, we arrive at the strong convergence in $L^{r}(0, T ; H)$ for any $r \in[1, \infty)$.

The strong convergence of the linear interpolants $\hat{u}_{\ell}$ towards $u$ in $L^{r}(0, T ; H)$ for any $r \in[1, \infty)$ now follows from (3.7) together with the boundedness of $\left\{\hat{u}_{\ell}\right\}$ in $L^{\infty}(0, T ; H)$.

We now prove strong convergence by employing a priori estimates for the discrete time derivative (and requiring additional compactness of the embedding of $V$ in $H$ ).

Theorem 3.7 (Strong convergence II). Let $u_{0} \in H$ and $b \in L^{q}\left(0, T ; V^{*}\right)$ be given, assume $u_{\ell}^{0} \rightarrow u_{0}$ in $H$ as $\ell \rightarrow \infty$ and let (3.6) be satisfied. If $V$ is compactly embedded in $H$ and if there is a constant $c>0$ such that for all $\ell \in \mathbb{N}$

$$
\left\|P_{m_{\ell}}\right\|_{V \leftarrow V} \leq c
$$

then, in addition to the assertions of Theorem 3.3, $u_{\ell}$ and $\hat{u}_{\ell}$ converge strongly in $L^{r}(0, T ; H)$ for any $r \in[1, \infty)$ towards the exact solution $u$, and the sequence of time derivatives $\hat{u}_{\ell}^{\prime}$ converges weakly in $\mathscr{X}^{*}$ towards the time derivative $u^{\prime}$ of the exact solution.

REMARK 3.8. The assumption (3.12) is an additional approximation property of the underlying function spaces and is known to be satisfied in special situations by appropriate finite element approximations in $W^{1, p}(\Omega)$ with $L^{2}(\Omega)$ as the pivot space (see, e.g., Crouzeix \& Thomée [6]). We later give an example of a finite element approximation of the porous medium equation in our setting with $L^{p}(\Omega) \subset H^{-1}(\Omega)$ and show that it fulfills (3.12) (see Lemma 4.2). 
Proof. [of Theorem 3.7] Theorem 3.7 is an immediate consequence of the results of Theorem 3.3 together with the following results that hold in addition to Lemma 3.5.

Under the additional assumption (3.12), Theorem 3.2 provides the uniform boundedness of the sequence of time derivatives $\hat{u}_{\ell}^{\prime}$ in $\mathscr{X}^{*}$ since

$$
\left\|\hat{u}_{\ell}^{\prime}\right\|_{\mathscr{X}^{*}} \leq\left\|\hat{u}_{\ell}^{\prime}\right\|_{L^{q}\left(0, T ; V^{*}\right)}
$$

and since

$$
\left\|\hat{u}_{\ell}^{\prime}\right\|_{L^{q}\left(0, T ; V^{*}\right)}^{q}=\tau_{\ell} \sum_{n=1}^{N_{\ell}}\left\|\frac{u^{n}-u^{n-1}}{\tau_{\ell}}\right\|_{V^{*}}^{q} .
$$

It follows the weak convergence of a subsequence of the time derivatives $\hat{u}_{\ell}^{\prime}$ towards $u^{\prime}$ in $\mathscr{X}^{*}$.

If $V \stackrel{c}{\hookrightarrow} H$ then, in view of the Lions-Aubin theorem (see, e.g., [14, Thm. 5.1 on p. 58]), $\mathscr{W} \stackrel{c}{\hookrightarrow} L^{2}(0, T ; H)$, and because of $\mathscr{W} \hookrightarrow L^{\infty}(0, T ; H)$, we also have $\mathscr{W} \stackrel{c}{\hookrightarrow}$ $L^{r}(0, T ; H)$ for any $r \in[1, \infty)$. Since $\left\{\hat{u}_{\ell}\right\}$ is now bounded in $\mathscr{W}$, we thus conclude with the strong convergence of a subsequence in $L^{r}(0, T ; H)$ for any $r \in[1, \infty)$. The limit can only be the weak limit $u$, i.e., the exact solution. Because of the uniqueness of the exact solution, again the whole sequence must converge.

The strong convergence of the piecewise constant interpolants follows from (3.7) and the boundedness of $\left\{u_{\ell}\right\}$ in $L^{\infty}(0, T ; H)$.

\section{Approximating $L^{p}(\Omega) \subset H^{-1}(\Omega)$}

In this section, we study a particular conforming finite element approximation in the one-dimensional case. So let $\Omega=(-L, L)$ for $L>0$. For $m \in \mathbb{N}$ given, let $\Omega$ be equidistantly partitioned into $M:=2^{m}$ subintervals $\left(x_{j-1}, x_{j}\right]$ with $x_{j}=-L+j h$ $(h=2 L / M, j=0,1, \ldots, M)$.

The finite element space $V_{m}$ now consists of all piecewise constant functions, i.e., $v \in V_{m}$ if and only if $v(x)=v_{j} \in \mathbb{R}$ for $x \in\left(x_{j-1}, x_{j}\right](j=1,2, \ldots, M)$. Besides the basis $\left\{\chi_{i}\right\}_{i=1}^{M}$ of $V_{m}$ given by the characteristic functions $\chi_{i}:=\chi_{\left(x_{i-1}, x_{i}\right]}$, we propose to use the special basis $\left\{\phi_{i}\right\}_{i=1}^{M}$ given by

$$
\begin{aligned}
& \phi_{1}=\frac{3}{2} \chi_{1}-\frac{1}{2} \chi_{2}, \quad \phi_{i}=-\frac{1}{2} \chi_{i-1}+\chi_{i}-\frac{1}{2} \chi_{i+1}(i=2,3, \ldots, M-1), \\
& \phi_{M}=-\frac{1}{2} \chi_{M-1}+\frac{3}{2} \chi_{M} .
\end{aligned}
$$

Note the different definitions for $\phi_{1}$ and $\phi_{M}$.

The advantage of this second basis lies in the fact that the basis function $\phi_{i}$ as well as the solution $(-\Delta)^{-1} \phi_{i}$ to the corresponding homogeneous Dirichlet problem both have small support. This leads to a tridiagonal structure of both the stiffness and mass matrix. To be precise, there holds

$$
\operatorname{supp}(-\Delta)^{-1} \phi_{i}=\operatorname{supp} \phi_{i}=\left[x_{i-2}, x_{i+1}\right], \quad i=2,3, \ldots, M-1,
$$

with obvious modifications for $i=1$ and $i=M$. Let

$$
\psi_{i}:=(-\Delta)^{-1} \phi_{i}
$$

A straightforward calculation employing Green's function shows that

$$
\psi_{1}(x)= \begin{cases}-\frac{3}{4}\left(x-x_{0}\right)^{2}+h\left(x-x_{0}\right) & \text { if } x \in\left[x_{0}, x_{1}\right] \\ \frac{1}{4}\left(x-x_{1}\right)^{2}-\frac{h}{2}\left(x-x_{1}\right)+\frac{h^{2}}{4} & \text { if } x \in\left(x_{1}, x_{2}\right] \\ 0 & \text { otherwise }\end{cases}
$$




$$
\psi_{i}(x)= \begin{cases}\frac{1}{4}\left(x-x_{i-2}\right)^{2} & \text { if } x \in\left(x_{i-2}, x_{i-1}\right] \\ -\frac{1}{2}\left(x-x_{i-1}-\frac{h}{2}\right)^{2}+\frac{3 h^{2}}{8} & \text { if } x \in\left(x_{i-1}, x_{i}\right] \\ \frac{1}{4}\left(x_{i+1}-x\right)^{2} & \text { if } x \in\left(x_{i}, x_{i+1}\right] \\ 0 & \text { otherwise }\end{cases}
$$

for $i=2,3, \ldots, M-1$, and

$$
\psi_{M}(x)= \begin{cases}\frac{1}{4}\left(x_{M-1}-x\right)^{2}-\frac{h}{2}\left(x_{M-1}-x\right)+\frac{h^{2}}{4} & \text { if } x \in\left(x_{M-2}, x_{M-1}\right] \\ -\frac{3}{4}\left(x_{M}-x\right)^{2}+h\left(x_{M}-x\right) & \text { if } x \in\left[x_{M-1}, x_{M}\right] \\ 0 & \text { otherwise }\end{cases}
$$

see also figure 4.1. Note that $\psi_{i} \in \mathscr{C}^{1}(\bar{\Omega})$ for $i=1,2, \ldots, M$.
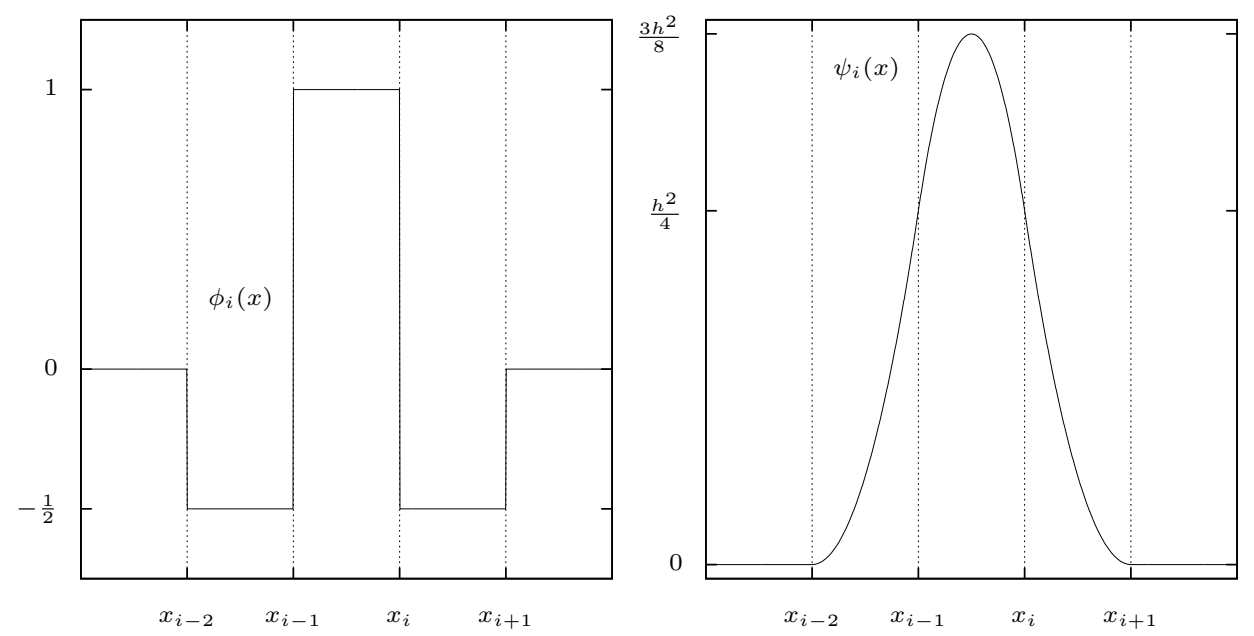

FIG. 4.1. Special piecewise constant basis function $\phi_{i}$ and its Dirichlet solution $\psi_{i}:=(-\Delta)^{-1} \phi_{i}$

We first analyze some approximation properties of our finite element spaces.

Lemma 4.1. The sequence $\left\{V_{m}\right\}_{m \in \mathbb{N}}$ of finite element spaces is a Galerkin scheme for $V=L^{p}(\Omega)$.

Proof. Obviously, we have $V_{m} \subset V_{m+1} \subset V$. The limited completeness immediately follows from the following estimate of the approximation error together with the density of $W^{1, p}(\Omega)$ in $L^{p}(\Omega)$.

Let the restriction operator $R_{m}: V \rightarrow V_{m}$ be defined by

$$
\left(R_{m} v\right)(x):=\frac{1}{h} \int_{x_{j-1}}^{x_{j}} v(\xi) d \xi \text { for } x \in\left(x_{j-1}, x_{j}\right](j=1,2, \ldots, M) .
$$


Then there holds for any $v \in W^{1, p}(\Omega)$

$$
\left\|v-R_{m} v\right\|_{0, p} \leq h\left\|v^{\prime}\right\|_{0, p} .
$$

This is seen as follows: By definition, we have with Hölder's inequality that

$$
\begin{aligned}
\left\|v-R_{m} v\right\|_{0, p}^{p} & =\sum_{j=1}^{M} \int_{x_{j-1}}^{x_{j}}\left|v(x)-\frac{1}{h} \int_{x_{j-1}}^{x_{j}} v(\xi) d \xi\right|^{p} d x \\
& \leq \sum_{j=1}^{M} \int_{x_{j-1}}^{x_{j}}\left(\frac{1}{h} \int_{x_{j-1}}^{x_{j}}|v(x)-v(\xi)| d \xi\right)^{p} d x \\
& \leq \frac{1}{h} \sum_{j=1}^{M} \int_{x_{j-1}}^{x_{j}} \int_{x_{j-1}}^{x_{j}}|v(x)-v(\xi)|^{p} d \xi d x \\
& \leq h^{p / q-1} \sum_{j=1}^{M} \int_{x_{j-1}}^{x_{j}} \int_{x_{j-1}}^{x_{j}} \int_{x_{j-1}}^{x_{j}}\left|v^{\prime}(\zeta)\right|^{p} d \zeta d \xi d x \\
& =h^{p}\left\|v^{\prime}\right\|_{0, p}^{p} .
\end{aligned}
$$

For every $v \in W^{1, p}(\Omega)$, we therefore obtain

$$
\inf _{v_{h} \in V_{m}}\left\|v-v_{h}\right\|_{0, p} \leq\left\|v-R_{m} v\right\|_{0, p} \leq h\left\|v^{\prime}\right\|_{0, p} .
$$

Since $W^{1, p}(\Omega)$ is dense in $V$, we have for every $v \in V$

$$
\inf _{v_{h} \in V_{m}}\left\|v-v_{h}\right\|_{0, p} \rightarrow 0 \text { as } m \rightarrow \infty,
$$

which is the limited completeness.

In view of the strong convergence result in Theorem 3.7, we shall now prove that the finite element approximation above possesses the property (3.12), i.e., that the $H^{-1}(\Omega)$-orthogonal projection onto the finite dimensional space of piecewise constant functions is $L^{p}(\Omega)$-stable.

Due to different Lebesgue exponents $\left(2\right.$ for $H^{-1}(\Omega)$ but $p$ for $L^{p}(\Omega)$ ) and the strongly nonlocal character of the $H^{-1}(\Omega)$ norm, the proof cannot be based upon an estimate of the approximation order of the restriction together with an inverse inequality. Instead, we directly calculate the projection belonging to our two different bases. This result is of interest for its own and is, to the best knowledge of the authors, the first result in this direction for $H^{-1}(\Omega)$-orthogonal projections.

Lemma 4.2. The sequence $\left\{V_{m}\right\}_{m \in \mathbb{N}}$ of finite element spaces fulfills (3.12), i.e, the $H^{-1}(\Omega)$-orthogonal projection onto the space of piecewise constant finite elements is $L^{p}(\Omega)$-stable.

Proof. As previously, we denote the $H^{-1}$-orthogonal projection onto $V_{m}$ by $P_{m}$. For arbitrary $v \in V=L^{p}(\Omega)$, we find with the restriction (4.4)

$$
\left\|P_{m} v\right\|_{0, p} \leq\left\|P_{m} v-R_{m} v\right\|_{0, p}+\left\|R_{m} v\right\|_{0, p} .
$$

With Hölder's inequality, we immediately find

$$
\left\|R_{m} v\right\|_{0, p}=\left(\sum_{k=1}^{M} \int_{x_{k-1}}^{x_{k}}\left|\frac{1}{h} \int_{x_{k-1}}^{x_{k}} v(\xi) d \xi\right|^{p} d x\right)^{1 / p} \leq\|v\|_{0, p} .
$$


Moreover, $P_{m} R_{m} v=R_{m} v$ for all $v \in L^{p}(\Omega)$. We thus find

$$
\left\|P_{m} v\right\|_{0, p} \leq\left\|P_{m}\left(v-R_{m} v\right)\right\|_{0, p}+\|v\|_{0, p},
$$

and it remains to prove that $\left\|P_{m}\left(v-R_{m} v\right)\right\|_{0, p} \leq c\|v\|_{0, p}$.

Let $z=P_{m}\left(v-R_{m} v\right) \in V_{m}$. Then $z$ is uniquely determined by

$$
(z, w)_{-1,2}=\left(v-R_{m} v, w\right)_{-1,2} \quad \forall w \in V_{m} .
$$

Since $z=\sum_{j=1}^{M} z_{j} \chi_{j}$ for some vector $\boldsymbol{z}:=\left[z_{1}, \ldots, z_{M}\right]^{T} \in \mathbb{R}^{M},(4.5)$ is equivalent to the linear system

$$
\sum_{j=1}^{M}\left(\chi_{j}, \phi_{i}\right)_{-1,2} z_{j}=\left(v-R_{m} v, \phi_{i}\right)_{-1,2}, \quad i=1,2, \ldots, M .
$$

Here we have used our first basis as trial but our second basis as test functions. Because of (2.1) and together with $\psi_{i}:=(-\Delta)^{-1} \phi_{i}$ (recall (4.3)), we see that (4.6) is equivalent to the linear system

$$
\boldsymbol{G} \boldsymbol{z}=\boldsymbol{y}, \quad \text { where } \boldsymbol{G}_{i j}=\left(\psi_{i}, \chi_{j}\right)_{0,2}, y_{i}=\left(v-R_{m} v, \psi_{i}\right)_{0,2} .
$$

A straightforward calculation shows that

$$
\boldsymbol{G}_{i j}=\int_{x_{j-1}}^{x_{j}} \psi_{i}(x) d x=\frac{h^{3}}{12} \begin{cases}3 & \text { if } j=i=1 \text { or } j=i=M \\ 1 & \text { if } j=i-1, \\ 4 & \text { if } j=i \text { and } i \neq 1 \text { and } i \neq M \\ 1 & \text { if } j=i+1 \\ 0 & \text { otherwise. }\end{cases}
$$

Denoting by $\|\cdot \mid\|_{r}(r \in[1, \infty])$ the usual $r$-vector norm on $\mathbb{R}^{M}$ as well as the induced matrix norm, we now find

$$
\left\|P_{m}\left(v-R_{m} v\right)\right\|_{0, p}=\|z\|_{0, p}=\left(h \sum_{i=1}^{M}\left|z_{i}\right|^{p}\right)^{1 / p}=h^{1 / p}\|\boldsymbol{z}\|_{p} \leq h^{1 / p}\left\|\boldsymbol{G}^{-1}\right\|_{p}\|\boldsymbol{y}\|_{p} .
$$

Since $\boldsymbol{G}$ is symmetric, also $\boldsymbol{G}^{-1}$ is symmetric, and the maximum row and column sum norms coincide. By interpolation (Riesz-Thorin theorem; see, e.g., Bergh \& Löfström [3, Thm. 1.1.1]), we find

$$
\left\|\boldsymbol{G}^{-1} \mid\right\|_{p} \leq\left\|\boldsymbol{G}^{-1}\right\|_{1}^{1 / p}\left\|\boldsymbol{G}^{-1}\right\|_{\infty}^{1 / q}=\left\|\boldsymbol{G}^{-1}\right\|_{\infty} .
$$

Since $\boldsymbol{G}$ is strictly diagonal dominant, the $\infty$-norm of $\boldsymbol{G}^{-1}$ can easily be estimated (see, e.g., Plato [18, Lemma 2.13 on p. 32]), and we obtain

$$
\left\|\boldsymbol{G}^{-1}\right\|_{\infty} \leq 6 h^{-3}
$$

and thus

$$
\left\|\boldsymbol{G}^{-1}\right\|_{p} \leq\left\|\boldsymbol{G}^{-1}\right\|_{\infty} \leq 6 h^{-3}
$$


We now estimate $\|\boldsymbol{y}\|_{p}$. Since with (4.3) and (4.4) for $i=2,3, \ldots, M-1$

$$
\begin{aligned}
y_{i}= & \left(v-R_{m} v, \psi_{i}\right)_{0,2}=\frac{1}{4} \int_{x_{i-2}}^{x_{i-1}}\left(v(x)-\frac{1}{h} \int_{x_{i-2}}^{x_{i-1}} v(\xi) d \xi\right)\left(x-x_{i-2}\right)^{2} d x \\
& +\int_{x_{i-1}}^{x_{i}}\left(v(x)-\frac{1}{h} \int_{x_{i-1}}^{x_{i}} v(\xi) d \xi\right)\left(-\frac{1}{2}\left(x-x_{i-1}-\frac{h}{2}\right)^{2}+\frac{3 h^{2}}{8}\right) d x \\
& +\frac{1}{4} \int_{x_{i}}^{x_{i+1}}\left(v(x)-\frac{1}{h} \int_{x_{i-2}}^{x_{i-1}} v(\xi) d \xi\right)\left(x_{i+1}-x\right)^{2} d x \\
= & \frac{1}{4} \int_{x_{i-2}}^{x_{i-1}} v(x)\left(\left(x-x_{i-2}\right)^{2}-\frac{h^{2}}{3}\right) d x \\
& +\int_{x_{i-1}}^{x_{i}} v(x)\left(-\frac{1}{2}\left(x-x_{i-1}-\frac{h}{2}\right)^{2}+\frac{3 h^{2}}{8}-\frac{h^{2}}{3}\right) d x \\
& +\frac{1}{4} \int_{x_{i}}^{x_{i+1}} v(x)\left(\left(x_{i+1}-x\right)^{2}-\frac{h^{2}}{3}\right) d x,
\end{aligned}
$$

we obtain with Hölder's inequality

$$
\left|y_{i}\right| \leq \operatorname{ch}^{2} \int_{x_{i-2}}^{x_{i+1}}|v(x)| d x \leq c h^{2+1 / q}\left(\int_{x_{i-2}}^{x_{i+1}}|v(x)|^{p} d x\right)^{1 / p},
$$

with obvious modifications for $i=1$ and $i=M$. It immediately follows that

$$
\|\boldsymbol{y}\|_{p} \leq c h^{2+1 / q}\|v\|_{0, p} .
$$

Altogether, we thus have from (4.8), (4.9), (4.10)

$$
\left\|P_{m}\left(v-R_{m} v\right)\right\|_{0, p} \leq c h^{1 / p-3+2+1 / q}\|v\|_{0, p}=c\|v\|_{0, p},
$$

which finally proves the assertion.

Finally, we can state a result about the convergence of the full discretization. We only focus on the piecewise constant in time approximation; results for the piecewise linear in time approximation can readily be gathered from Theorems 3.3, 3.6, and 3.7. Note here that the assumption (3.6) may lead to a coupling of the time step and spatial mesh size, especially for rough initial data.

Corollary 4.3. Let $\Omega=(-L, L)$ and let $u_{0} \in H^{-1}(\Omega), f \in L^{q}\left(0, T ;\left(W^{2, p}(\Omega) \cap\right.\right.$ $\left.\left.W_{0}^{1, p}(\Omega)\right)^{*}\right), g \in L^{q}\left(0, T ;\left(W^{1 / q, p}(\partial \Omega)\right)^{*}\right)$ be given. Let (1.1) be approximated by the piecewise constant finite element method combined with the backward Euler method as described above. The piecewise constant in time and space numerical solution then converges weakly in $L^{p}(\Omega \times(0, T))$ and weakly* in $L^{\infty}\left(0, T ; H^{-1}(\Omega)\right)$ towards the exact very weak solution as the time step and mesh size tend to zero.

If one of the stronger monotonicity assumptions (1.3) or (1.4) are fulfilled then the numerical solution converges also strongly in $L^{p}(\Omega \times(0, T))$ and in $L^{r}\left(0, T ; H^{-1}(\Omega)\right)$ for any $r \in[1, \infty)$.

If (3.6) holds then the numerical solution converges strongly in $L^{r}\left(0, T ; H^{-1}(\Omega)\right)$ for any $r \in[1, \infty)$. 
Proof. The weak convergence of the numerical solution immediately results from Theorem 3.3 applied to the very weak formulation of (1.1) with $V=L^{p}(\Omega)$ and $H=$ $H^{-1}(\Omega)$. Note in particular that $L^{p}(0, T ; V)=L^{p}(\Omega \times(0, T))$ for $V=L^{p}(\Omega)$ and recall that $f, g$ lead to the right-hand side $b \in L^{q}\left(0, T ; V^{*}\right)$ in the abstract formulation.

The strong convergence in case of one of the stronger monotonicity assumptions (1.3) or (1.4) is a direct consequence of Theorem 3.6.

If (3.6) is fulfilled then strong convergence follows from Theorem 3.7 together with Lemma 4.2, which shows that assumption (3.12) is satisfied. Recall here that, in the one-dimensional case, $L^{p}(\Omega)$ is compactly embedded in $H^{-1}(\Omega)$ for any $p \in \Pi$. $\square$

For a discussion of a possible step size restriction due to (3.6), see Remark 5.1.

\section{Numerical results for the Barenblatt solution}

We use the notation of the preceding section. In particular, recall that we are working with two types of basis functions: $\chi_{i}:=\chi_{\left(x_{i-1}, x_{i}\right]}$ and $\phi_{i}$, which are given by (4.1). Recall that the matrix $\boldsymbol{G}$ is given by (4.7). For all $n=0,1, \ldots, N$, let $u^{n} \in V_{m}$

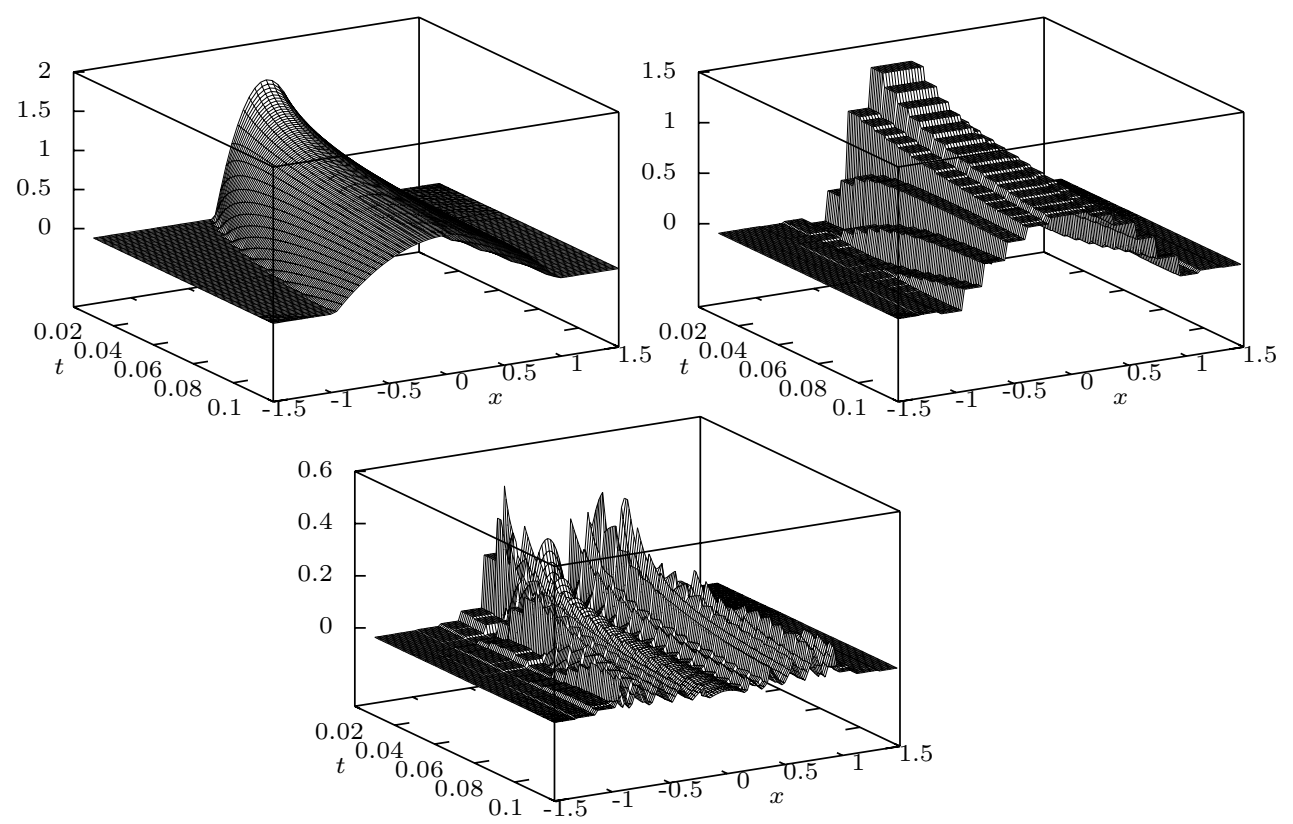

FIG. 5.1. Exact solution, numerical solution, and the respective errors for $p=3$ on the interval $\Omega=(-1.5,1.5)$, using 16 finite elements and time steps. Vertical axes have different scales.

be given by

$$
u^{n}(x)=\sum_{i=1}^{M} u_{i}^{n} \phi_{i}(x), \quad x \in[-L, L]
$$

As test functions, we take the basis functions $\chi_{i}(i=1,2, \ldots, M)$. Let $\boldsymbol{u}^{n}:=$ $\left[u_{1}^{n}, \ldots, u_{M}^{n}\right]^{T}$. The numerical scheme (3.1) then is equivalent to

$$
\boldsymbol{G} \frac{\boldsymbol{u}^{n}-\boldsymbol{u}^{n-1}}{\tau}+\boldsymbol{A}\left(\boldsymbol{u}^{n}\right)=\boldsymbol{b}^{n}, \quad n=1,2, \ldots, N,
$$




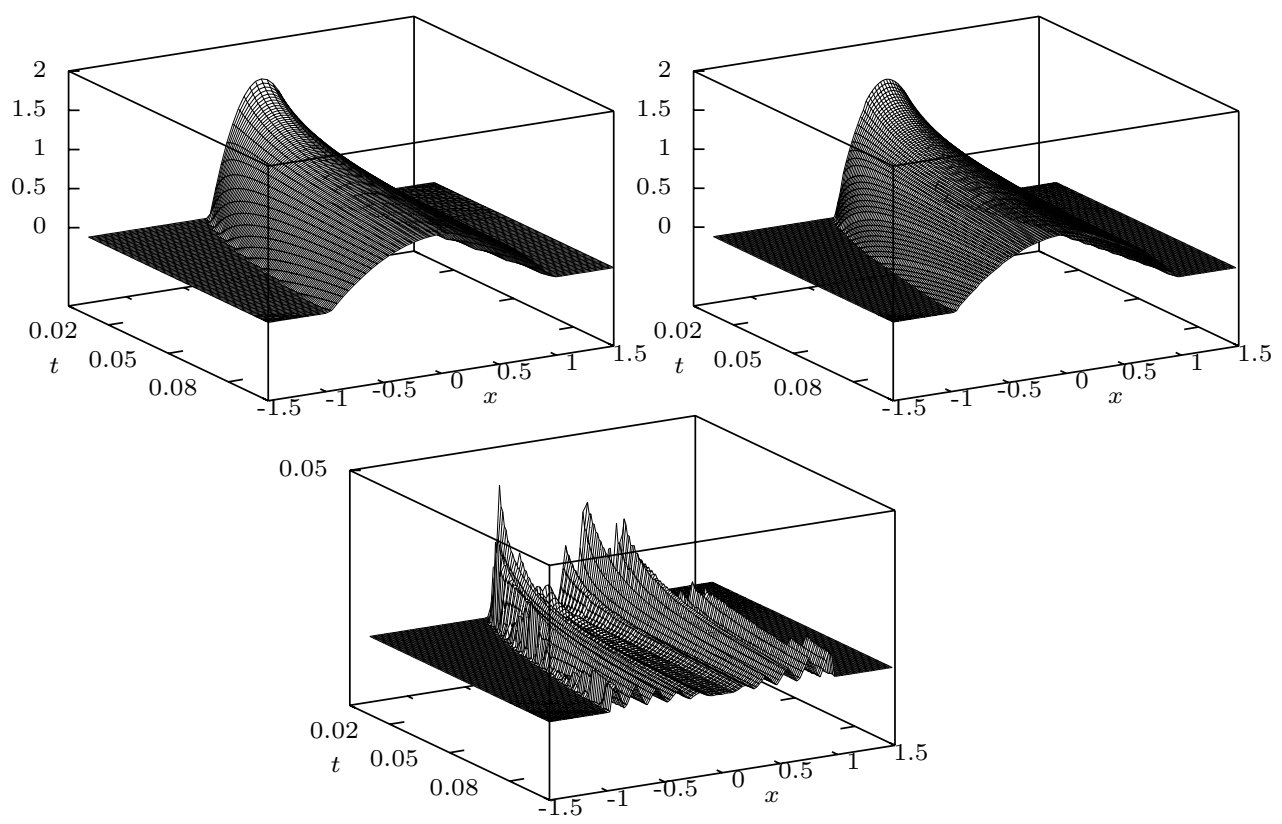

FIG. 5.2. Exact solution, numerical solution, and the respective errors for $p=3$ on the interval $\Omega=(-1.5,1.5)$, using 256 finite elements and 1024 time steps. Vertical axes have different scales.

where

$$
\boldsymbol{A}\left(\boldsymbol{u}^{n}\right):=\left[\alpha_{1}^{n}, \ldots, \alpha_{M}^{n}\right]^{T} \quad \text { with } \quad \alpha_{j}^{n}:=\int_{x_{j-1}}^{x_{j}} \alpha\left(\sum_{i=1}^{M} u_{i}^{n} \phi_{i}(x)\right) d x
$$

so that

$$
\begin{aligned}
& \alpha_{1}^{n}=h \alpha\left(\frac{3}{2} u_{1}^{n}-\frac{1}{2} u_{2}^{n}\right), \\
& \alpha_{j}^{n}=h \alpha\left(-\frac{1}{2} u_{j-1}^{n}+u_{j}^{n}-\frac{1}{2} u_{j+1}^{n}\right) \text { for } j=2,3, \ldots, M-1, \\
& \alpha_{M}^{n}=h \alpha\left(-\frac{1}{2} u_{M-1}^{n}+\frac{3}{2} u_{M}^{n}\right) .
\end{aligned}
$$

Moreover, $\boldsymbol{u}^{0}$ and

$$
\boldsymbol{b}^{n}:=\left[b_{1}^{n}, \ldots, b_{M}^{n}\right]^{T} \quad \text { with } \quad b_{j}^{n}:=\int_{x_{j-1}}^{x_{j}} b^{n}(x) d x
$$

are given. We recall here that $\boldsymbol{G}$ is symmetric.

If we take $\chi_{i}(i=1,2, \ldots, M)$ as trial and $\phi_{i}(i=1,2, \ldots, M)$ as test functions, the resulting numerical scheme reads as

$$
\boldsymbol{G} \frac{\tilde{\boldsymbol{u}}^{n}-\tilde{\boldsymbol{u}}^{n-1}}{\tau}+\tilde{\boldsymbol{A}}\left(\tilde{\boldsymbol{u}}^{n}\right)=\tilde{\boldsymbol{b}}^{n}, \quad n=1,2, \ldots, N,
$$



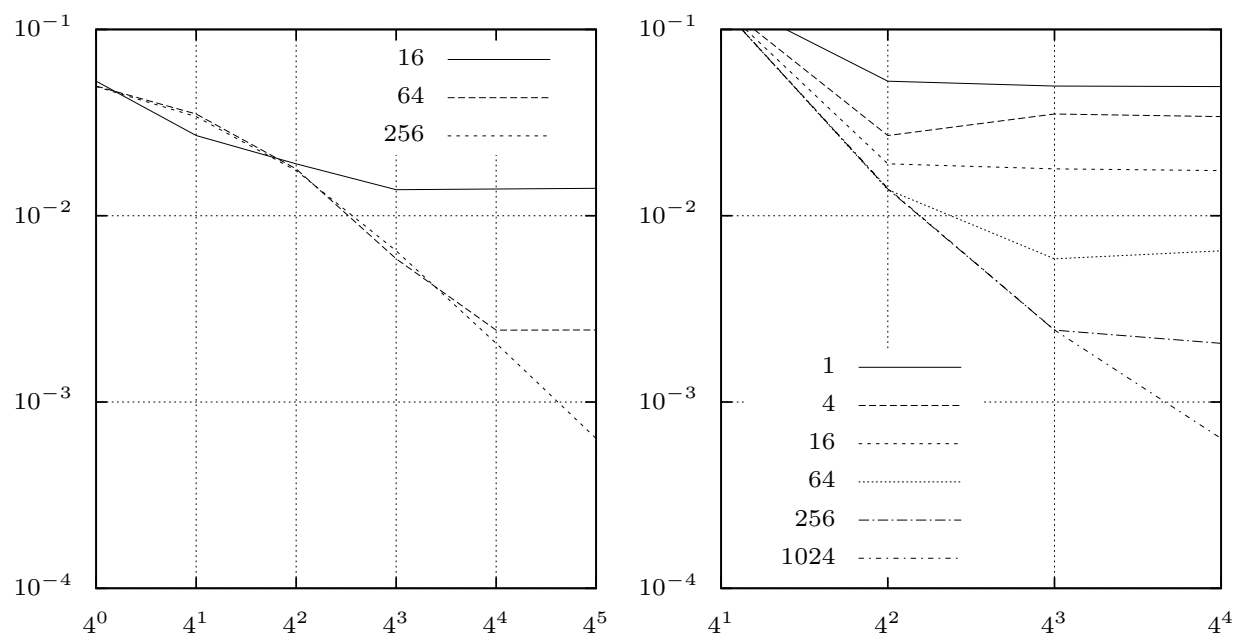

FIG. 5.3. Plot of convergence of the numerical solution to the Barenblatt solution for $p=3$, error $u-u_{M, N}$ is measured in the $L^{3}((-1.5,1.5) \times(0.01,0.11))$ norm. The scales are logarithmic. Different lines correspond to different numbers of finite elements (from 16 to 256) on the left and different numbers of time steps (from 1 to 1024) on the right.

where now $\tilde{\boldsymbol{u}}:=\left[\tilde{u}_{1}^{n}, \ldots, \tilde{u}_{M}^{n}\right]^{T}$ such that

$$
u^{n}(x)=\sum_{i=1}^{M} \tilde{u}_{i}^{n} \chi_{i}(x), \quad x \in[-L, L]
$$

with $\tilde{\boldsymbol{u}}^{0}$ given. Moreover, we have $\tilde{\boldsymbol{A}}\left(\tilde{\boldsymbol{u}}^{n}\right):=\left[\tilde{\alpha}_{1}^{n}, \ldots, \tilde{\alpha}_{M}^{n}\right]^{T}$ with

$$
\begin{aligned}
& \tilde{\alpha}_{1}^{n}=\frac{3 h}{2} \alpha\left(\tilde{u}_{1}^{n}\right)-\frac{h}{2} \alpha\left(\tilde{u}_{2}^{n}\right), \\
& \tilde{\alpha}_{j}^{n}=-\frac{h}{2} \alpha\left(\tilde{u}_{j-1}^{n}\right)+h \alpha\left(\tilde{u}_{j}^{n}\right)-\frac{h}{2} \alpha\left(\tilde{u}_{j+1}^{n}\right) \quad \text { for } j=2,3, \ldots, M-1, \\
& \tilde{\alpha}_{M}^{n}=-\frac{h}{2} \alpha\left(\tilde{u}_{M-1}^{n}\right)+\frac{3 h}{2} \alpha\left(\tilde{u}_{M}^{n}\right) .
\end{aligned}
$$

Finally, we have $\tilde{\boldsymbol{b}}^{n}:=\left[\tilde{b}_{1}^{n}, \ldots, \tilde{b}_{M}^{n}\right]^{T}$ with

$$
\begin{aligned}
& \tilde{b}_{1}^{n}:=\frac{3}{2} \int_{x_{0}}^{x_{1}} b^{n}(x) d x-\frac{1}{2} \int_{x_{1}}^{x_{2}} b^{n}(x) d x \\
& \tilde{b}_{j}^{n}:=-\frac{1}{2} \int_{x_{j-2}}^{x_{j-1}} b^{n}(x) d x+\int_{x_{j-1}}^{x_{j}} b^{n}(x) d x-\frac{1}{2} \int_{x_{j}}^{x_{j+1}} b^{n}(x) d x \text { for } j=2,3, \ldots, M-1, \\
& \tilde{b}_{M}^{n}:=-\frac{1}{2} \int_{x_{M-2}}^{x_{M-1}} b^{n}(x) d x+\frac{3}{2} \int_{x_{M-1}}^{x_{M}} b^{n}(x) d x .
\end{aligned}
$$


The mass matrix here is again $\boldsymbol{G}$ since if $\boldsymbol{M}$ denotes the matrix for the basis transformation then $\boldsymbol{G}=\boldsymbol{M} \boldsymbol{G} \boldsymbol{M}^{-1}$.

Note that in both the cases, the unknown coefficient vector can be determined step-by-step. In what follows, we restrict our considerations to the second representation.

We now describe our test problem. Let $u_{0}$ be the $\delta$-distribution, which is indeed an element of $H^{-1}(\Omega)$ since $\Omega$ is one-dimensional. Moreover, let $f=g=0$ such that $b=0$. Non-zero boundary values $g$ or right-hand side $f$ would lead to a non-zero right-hand side $b$ in the very weak formulation. Finally, we consider $\alpha(z)=|z|^{p-2} z$ and restrict our considerations to the case $p=3$.

The exact (very weak) solution then is the Barenblatt solution given by

$$
u(x, t)=t^{-1 / p}\left(C-\frac{p-2}{2 p(p-1)} x^{2} t^{-2 / p}\right)_{+}^{1 /(p-2)},
$$

where $(\cdot)_{+}$denotes the positive part of a real number and where $C>0$ is chosen such that

$$
\int_{\Omega} u(t, x)=1, \quad \text { i.e., } \quad C=\frac{\sqrt[3]{3}}{4} \text { for } p=3 .
$$

See, e.g., Vázquez [23], for a multidimensional formula and further details.

On the current grid, the initial condition is approximated by

$$
u^{0}(x)=\frac{1}{2 h}\left\{\begin{array}{l}
1 \quad \text { for } x \in\left(x_{M / 2-1}, x_{M / 2+1}\right] \\
0 \quad \text { otherwise }
\end{array}\right.
$$

such that $\tilde{\boldsymbol{u}}^{0}=[0, \ldots, 0,1,1,0, \ldots, 0]^{T} / 2 h$ and $\boldsymbol{u}^{0}=\boldsymbol{M} \tilde{\boldsymbol{u}}^{0}$.

We now describe and present the results of numerical tests. We choose the domain $(-1.5,1.5) \times(0,0.11)$ and solve the approximation $(5.1)$. In each time step, we solve the corresponding nonlinear problem using Newton's iterative method. We present the output of the numerical calculation, the exact solution and the difference between the exact solution and the numerical calculation as 3D plots in figures 5.1 and 5.2. The time range of the plot is $[0.01,0.11]$.

Let us denote by $u_{M, N}$ the piecewise constant interpolation of the numerical solution for the time grid with $N$ subintervals and the spatial grid with $M$ subintervals. We calculate the error $u-u_{M, N}$ in the $L^{3}((-1.5,1.5) \times(0.01,0.11))$ norm. Note that while the scheme runs well when started from an approximation of the $\delta$-distribution, the exact solution cannot be well resolved on the computer for very small values of $t$ since $u_{0}=\delta$. This is the reason for starting the error measurement slightly away from zero.

Due to Corollary 4.3, we have strong convergence in $L^{3}(\Omega \times(0, T))$ since the uniform monotonicity condition (1.4) is satisfied. Numerical tests illustrate and support the theoretical results; see figure 5.3 and table 5.1.

Notice that we do not need the coupling of time and space grid sizes (3.6) in order to get the strong convergence of the piecewise constant interpolation. Nevertheless, with respect to the condition (3.6) in combination with the $\delta$-distribution as the initial datum, we have the following remark.

REMARK 5.1. Assumption (3.6) in general means a coupling of the time step and spatial mesh size. Let $u_{0}=\delta$ be approximated as above. Then

$$
\frac{\tau}{h^{p-1}} \leq c
$$




\begin{tabular}{|c||c|c|c|c|}
\hline$N \Downarrow \quad M \Rightarrow$ & $\mathbf{4}$ & $\mathbf{1 6}$ & $\mathbf{6 4}$ & $\mathbf{2 5 6}$ \\
\hline \hline $\mathbf{1}$ & $1.5 \mathrm{E}-01$ & $5.3 \mathrm{E}-02$ & $5.0 \mathrm{E}-02$ & $4.9 \mathrm{E}-02$ \\
\hline $\mathbf{4}$ & $1.4 \mathrm{E}-01$ & $2.7 \mathrm{E}-02$ & $3.5 \mathrm{E}-02$ & $3.4 \mathrm{E}-02$ \\
\hline $\mathbf{1 6}$ & $1.3 \mathrm{E}-01$ & $1.9 \mathrm{E}-02$ & $1.8 \mathrm{E}-02$ & $1.8 \mathrm{E}-02$ \\
\hline $\mathbf{6 4}$ & $1.3 \mathrm{E}-01$ & $1.4 \mathrm{E}-02$ & $5.9 \mathrm{E}-03$ & $6.5 \mathrm{E}-03$ \\
\hline $\mathbf{2 5 6}$ & $1.3 \mathrm{E}-01$ & $1.4 \mathrm{E}-02$ & $2.4 \mathrm{E}-03$ & $2.1 \mathrm{E}-03$ \\
\hline $\mathbf{1 0 2 4}$ & $1.3 \mathrm{E}-01$ & $1.4 \mathrm{E}-02$ & $2.4 \mathrm{E}-03$ & $6.4 \mathrm{E}-04$ \\
\hline
\end{tabular}

TABLE 5.1. Table of convergence for errors $u-u_{M, N}$ measured in the $L^{3}((-1.5,1.5) \times$ $(0.01,0.11))$ norm. Each column quarters the spatial mesh size $h$ and each row quarters the time step $\tau$.

is required for satisfying (3.6), which is rather a severe restriction.

Acknowledgment. The authors would like to thank Eskil Hansen for helpful discussions regarding the subject of the paper.

\section{REFERENCES}

[1] V. Barbu, Nonlinear Semigroups and Differential Equations in Banach Spaces, Noordhoff Int. Publ., Leyden, 1976.

[2] J. Barrett and P. Knabner, Finite element approximation of the transport of reactive solutes is porous media. Part 2: Error estimates for equilibrium adsorption processes, SIAM J. Numer. Anal., 34, 455-479, 1997.

[3] J. Bergh and J. Löfström, Interpolation Spaces, Springer, Berlin, 1976.

[4] W.J. Beyn, B. Gess, P. Lescot, and M. Röckner, The global random attractor for a class of stochastic porous media equations, Commun. Part. Differ. Eqs., 36(3), 446-469, 2010.

[5] H. Brézis, Analyse Fonctionnelle: Théorie et Applications, Dunod, Paris, 1999.

[6] M. Crouzeix and V. Thomée, The stability in $L_{p}$ and $W_{p}^{1}$ of the $L_{2}$-projection onto finite element function spaces, Math. Comput., 48(178), 521-532, 1987.

[7] C. Ebmeyer, Error estimates for a class of degenerate parabolic equations, SIAM J. Numer. Anal., 35, 1095-1112, 1998.

[8] E. Emmrich, Two-step BDF time discretisation of nonlinear evolution problems governed by monotone operators with strongly continuous perturbations, Comput. Meth. Appl. Math., $9(1), 37-62,2009$.

[9] E. Emmrich and M. Thalhammer, Stiffly accurate Runge-Kutta methods for nonlinear evolution problems governed by a monotone operator, Math. Comput., 79, 785-806, 2010.

[10] H. Fujita, N. Saito, and T. Suzuki, Operator Theory and Numerical Methods, Elsevier, Amsterdam, 2001.

[11] H. Gajewski, K. Gröger, and K. Zacharias, Nichtlineare Operatorgleichungen und OperatorDifferentialgleichungen, Akademie-Verlag, Berlin, 1974.

[12] D. Gilbarg and N.S. Trudinger, Elliptic Partial Differential Equations of Second Order, Springer, Berlin, 1998.

[13] E. Hansen and A. Ostermann, Finite element Runge-Kutta discretizations of porous medium type equations, SIAM J. Numer. Anal., 46, 1769-1779, 2008.

[14] J.L. Lions, Quelques Méthodes de Résolution des Problèmes aux Limites Non Linéaires, Dunod Gauthier-Villars, Paris, 1969.

[15] A. Mizutani, N. Saito, and T. Suzuki, Finite element approximation for degenerate parabolic equations. An application of nonlinear semigroup theory, ESAIM: M2AN Math. Mod. Numer. Anal., 39, 755-780, 2005.

[16] J. Nečas, Les méthodes directes en théorie des équations elliptiques, Masson, Paris, 1967.

[17] R. Nochetto and C. Verdi, Approximation of degenerate parabolic problems using a numerical integration, SIAM J. Numer. Anal., 25, 784-814, 1988.

[18] R. Plato, Concise Numerical Mathematics, Amer. Math. Soc., Providence, RI, 2003.

[19] I.S. Pop and W. Yong, A numerical approach to degenerate parabolic equations, Numer. Math., 92, 357-381, 2002.

[20] T. Roubíček, Nonlinear Partial Differential Equations with Applications, Birkhäuser, Basel, 2005.

[21] J. Rulla and N.J. Walkington, Optimal rates of convergence for degenerate parabolic problems in two dimensions, SIAM J. Numer. Anal., 33, 56-67, 1996. 
[22] R. Temam, Numerical Analysis, D. Reidel Publ. Comput., Dordrecht, 1973.

[23] J.L. Vázquez, The Porous Medium Equation: Mathematical Theory, Oxford Univ. Press, Oxford, 2007.

[24] J. Wloka, Partial Differential Equations, Cambridge Univ. Press, Cambridge, 1987.

[25] E. Zeidler, Nonlinear Functional Analysis and its Applications, II/B: Nonlinear Monotone Operators, Springer, Berlin, 1990. 